\title{
Two-equation and multi-fluid turbulence models for Richtmyer-Meshkov mixing
}

loannis W. Kokkinakis, ${ }^{1,}$ a) Dimitris Drikakis, ${ }^{2,}$ b) and David L. Youngs ${ }^{1}$

1) Department of Mechanical \& Aerospace Engineering, University of Strathclyde, Glasgow, G1 1XJ, UK

${ }^{2)}$ University of Nicosia, Nicosia, CY-2417, Cyprus

(Dated: 9 July 2020)

This paper concerns an investigation of two different approaches in modelling the turbulent mixing induced by the Richtmyer-Meshkov instability (RMI): A two-equation $K-L$ multi-component Reynolds-Averaged NavierStokes (RANS) model and a two-fluid model. We have improved the accuracy of the $K$ - $L$ model by implementing new modifications, including a realizability condition for the Reynolds stress tensor and a threshold in the production of the turbulence kinetic energy. We examine the models in one-dimensional (1D) form in the (re)-shocked mixing of a double-planar air and sulfur-hexafluoride $\left(\mathrm{SF}_{6}\right)$ interfaces of Atwood number $\left|A_{t}\right| \simeq 0.6853$. Furthermore, we investigated the models' accuracy to RMI-induced mixing of a (re)-shocked planar-inverse chevron air- $\mathrm{SF}_{6}$ interface. Relevant integral quantities in time, as well as instantaneous profiles and contour plots, are used to assess the models' accuracy against high-resolution implicit Large Eddy Simulations (iLES). The proposed modifications improve the efficiency of the $K-L$ model. The model is designed as a simple model able to capture the self-similar growth of Rayleigh-Taylor and Richtmyer-Meshkov flows. The two-fluid model remains overall more accurate but is also computationally more expensive.

\section{INTRODUCTION}

The Richtmyer-Meshkov instability (RMI) is identified and examined in the works of Richtmyer ${ }^{1}$ and Meshkov $^{2}$ on impulsively accelerated interfaces between two fluid layers with different densities caused by an incident shock wave. Previous results suggest that at the early stages the instabilities develop rapidly and reach a non-linear state where the mixing layer between heavy and light components exhibits characteristic mushroomshaped bubbles and spikes. The bubble and spike features may later break-down into fully turbulent mixing regions of flow and are thus associated with transition and turbulent mixing. These features develop at an early stage due to random perturbations on the interface that quickly become amplified after the impulse-like acceleration arising from the passing shock wave. We can obtain an understanding behind the processes and mechanisms dictating their development by examining different amplitudes of the initial interface perturbations and the resulting mixing that develops.

The process mentioned above is of particular importance concerning inertial confinement fusion (ICF), where RMI-induced mixing is a critical factor in predicting the performance of ICF capsules $^{3-5}$. In ICF a powerful laser is used to compress a spherical capsule containing thermonuclear material ${ }^{4}$. During the implosion, RM instabilities at the interfaces between the light and heavy materials trigger turbulent mixing that not only dilutes the fuel but also cools it. This inhibits higher temperatures and compression of the fuel, significantly reducing the efficiency of the thermonuclear reaction. Hence we must advance the understanding of

\footnotetext{
a)Electronic mail: ioannis.kokkinakis@strath.ac.uk

b)Electronic mail: drikakis.d@unic.ac.cy
}

the instabilities mechanism. RMI-induced mixing is not only restricted to ICF. It spans across many scientific disciplines. Other examples include aeronautical engineering for supersonic combustion ramjet (SCRAMJET) propulsion $^{6}$, and astrophysics for the prediction of the evolution of supernovae ${ }^{7,8}$.

In most cases, large Reynolds numbers $(R e)$ are associated with instabilities that are broadband in nature. After the shock, the instabilities grow in a linear, selfsimilar manner but eventually become highly non-linear provided the duration is long enough. Once non-linear at a late time, the flow breaks down to fully turbulent. It has initially evolved self-similarly with an eddy length scale $(L)$ that grows in proportion to the amplitude of the mixing region $(h)$ during an early, transitional, period. From a computational perspective, high-resolution direct numerical simulations can resolve instabilities of a $R e<10^{4}$. However, for supernovae or other cases where the Reynolds number is typical $R e>10^{6}$, not all scales can be adequately resolved. Therefore Large Eddy Simulations (LES) or Reynolds-averaged Navier-Stokes (RANS) models are necessary to describe the effects of these unresolved structures such as the hydrodynamic RMI.

Over the last few decades, several turbulence models have been developed with the above in mind and are classified into three main categories. The simplest type of model describes the physical process by balancing inertia, buoyancy and drag forces during the formation and evolution of the mixing width. It leads to a set of ordinary differential equations and the models are referred to as buoyancy-drag models ${ }^{9-12}$. This type of model is useful for obtaining simple estimates of the mix widths in one dimensional flows but does not give detailed mix distributions in more complex flows such as those considered here.

These problems were addressed in the second category 
of models proposed, the so-called two-fluid (or multifluid) models ${ }^{13-16}$, and references therein. In addition to the mean flow equations, these models also solve a separate set for each fluid; therefore these are relatively complex, but give a good representation of the mixing processes. In particular, they can describe demixing processes and can also capture the relative motion of the different fluid fragments correctly.

Another class of models that are significantly simpler than two-fluid models maintains the individual species fraction, but assign a single fluctuating velocity representative of the mixture ${ }^{17-20}$. These models are known as two-equation turbulence models because they consist of evolutionary equations for the turbulence kinetic energy per unit mass and its dissipation rate ${ }^{17,18}$ or equivalent turbulence length scale ${ }^{19,20}$. Specifically, these models postulate a turbulent viscosity, a Reynolds stress, and dissipation terms, as well as buoyancy terms for modelling Rayleigh-Taylor (RT) and RM instabilities. This type of models is capable of handling multi-dimensional, multi-fluid processes under variable accelerations. However, they cannot capture demixing.

We investigate the two-equation $K-L^{19,20}$ and twofluid $(\mathrm{TF})^{13,21}$ turbulence models in the simulation of Richtmyer-Meshkov instability (RMI) induced mixing. Previously $^{19}$, the turbulence model coefficients were calibrated to accurately model the mixing width evolution of the Richtmyer-Meshkov, as well as Rayleigh-Taylor and Kelvin-Helmholtz instabilities, in one dimensional simulations based on experimental data. Here, we extend the application of the $K$ - $L$ turbulence model to two dimensions and examine the effect of an inclined interface. We use the the flow case of the inverse-chevron (IC) experiment by Holder and Barton ${ }^{22}$. The use of a laser-sheet technique in the experiments has shown that high resolution iLES provides a very good model of the experimental behavior and in the present paper iLES results are used for RANS model validation. Thus, by neglecting the viscous stresses the thin boundary layer does not have to be resolved, thereby significantly reducing the computational requirements and allowing for high-resolution iLES simulations $^{23,24}$.

We model the short surface wavelength perturbations by using different power-spectra proportional to the mode and the same standard deviation ${ }^{23,25,26}$. The above consequently introduces a three-dimensionality to the test case that promotes turbulent mixing to a varying degree in time as the interface interacts with the initial shock and generated reflections. While turbulence is inherently three dimensional (3D), mean flow quantities remain strongly two dimensional (2D). In other words, the notion is that the $3^{\text {rd }}$ direction develops fluxes only associated with the turbulent field and the mean flow properties behave as a quasi-two-dimensional case. The purpose of the experiments and high-resolution iLES simulations is to form a well defined two-dimensional flow case with fine-scale turbulent mixing superimposed on the interface. The $2 \mathrm{D}$ flow case is suitable for the validation of
2D Reynolds-Averaged Navier-Stokes turbulence models.

We first examine the accuracy of the models in a simplified version of the inverse-chevron case, in which both interfaces are planar, henceforth labelled as doubleplanar case. Consequently, similarly to the spanwise direction, the wall-normal direction becomes homogeneous too and averages can thus be reduced onto a onedimensional field, varying in space along the streamwise $x$-direction only. The above setting allows the examination of the turbulence models in the simplest, onedimensional, form without the presence of cross-terms affecting the solution.

We organise the paper as follows. Section §II provides a full description of the double-planar and inversechevron test-cases followed by a brief description of the iLES method in §III. Section §IV discusses the formulation of the TF (§IV A) and $K-L(\S I V B)$ mixing models used in the current study along with further details of any modifications introduced given in $\S I V B 1$. Next, $\S I V \mathrm{C}$ details the numerical methods as well as boundary and initial conditions used for the turbulence models. $\S \mathrm{V}$ presents the results obtained using the $K-L$ and twofluid mixing models. We compare the results against the high-resolution iLES results. Finally, §VI gives a detailed summary of the most important findings made.

\section{DESCRIPTION OF TEST-CASES}

Initially, all fluids are at rest apart from the shocked incoming air $\left(a i r^{\star}\right)$. Using shock tube relations, we calculate the Mach number of the shock wave $\left(M_{s}\right)$ :

$$
M_{s}=\sqrt{\frac{\gamma-1}{2 \gamma}+\frac{(\gamma+1) p_{\mathrm{air}^{\star}}}{2 \gamma p_{\mathrm{air}}}}
$$

The speed of the shock wave $\left(u_{s}\right)$ is then computed based on the speed of sound $\left(s_{\text {air }}\right)$ in the preshocked region. Hence:

$$
u_{s}=s_{\text {air }} M_{s}=\sqrt{\frac{\gamma p_{\text {air }}}{\rho_{\text {air }}}} M_{s}
$$

Finally, the speed of the compressed air after the shock wave is given by:

$$
u_{\mathrm{air}^{\star}}=\left(1-\frac{\rho_{\mathrm{air}}}{\rho_{\mathrm{air}}}\right) \mathrm{u}_{s}
$$

As an incident shock wave of $M_{s}>1$ passes from left to right, the membranes separating the two fluids rupture and Richtmyer-Meshkov instabilities begin to develop. The membranes are supported on a fine wire mesh with a grid spacing of $0.4 \mathrm{~cm}$. The initially small perturbations multiply in size, leading to high Reynolds number turbulent mixing of the two gases. The interface perturbations are further amplified by the formation of reflected waves as the primary, as well as subsequent shock waves that pass through the material interfaces. 


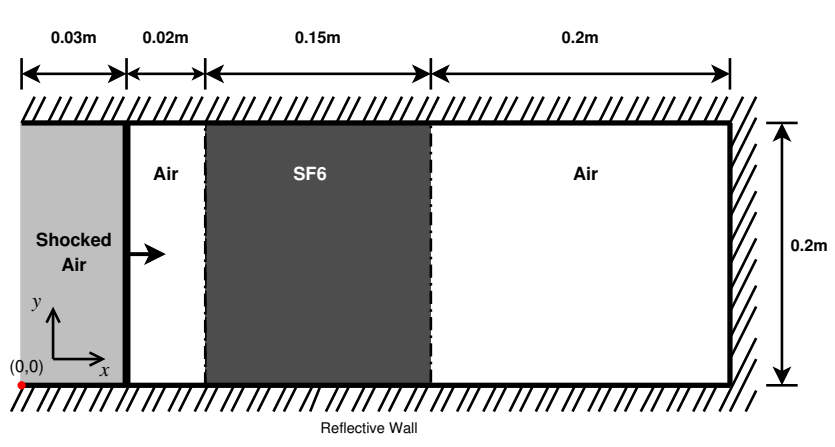

FIG. 1. Illustration of the double-planar (DP) boundary and initial conditions.

TABLE I. Initial fluid properties for the double-planar (DP) case at an initial Atwood number of $\left|A_{t}\right| \simeq 0.6853$.

\begin{tabular}{lccc}
\hline \hline & Shocked Air & Air & $\mathrm{SF}_{6}$ \\
\hline Density $\left(\mathrm{kg} / \mathrm{m}^{3}\right)$ & 1.7047 & 1.184 & 6.34 \\
Pressure $(\mathrm{Pa})$ & 167,500 & 100,000 & 100,000 \\
$\gamma$ & 1.4 & 1.4 & 1.076 \\
\hline \hline
\end{tabular}

Both the iLES and RANS assume high-Re behavior where the dissipation processes are controlled by the cascade to high wave-numbers and are not dependent on the Reynolds, Re, and Schmidt, $S c$, numbers. The present iLES is conducted assuming that the Reynolds number is high enough so that the flow is beyond the mixing transition, as defined by Dimotakis ${ }^{27}$, for the effect of the Schmidt number to become important. According to experimental results ${ }^{27,28}$ and references therein, the mixing transition corresponds to $R e \approx 10^{4}$. The iLES data used here apply to high-Re number mixing since the effective Reynolds number was previously ${ }^{24}$ found to be of order $10^{5}$.

A depiction of the initial condition and properties of the fluid variables are given in Fig. 1 and Table I, respectively. According to Eqs. (1)-(3), the following quantities are evaluated: $M_{s} \simeq 1.2564, u_{s} \simeq 432.036 \mathrm{~m} / \mathrm{s}$ and $u_{\mathrm{air}^{\star}} \simeq 131.965 \mathrm{~m} / \mathrm{s}$.

The inverse-chevron case considered is based on the experiment of Holder and Barton ${ }^{22}$, which features a block of dense sulfur-hexafluoride $\left(\mathrm{SF}_{6}\right)$ gas encased in air within a tube. A depiction of the initial condition and fluid properties are given in Fig. 2 and Table II, respectively. According to Eqs. (1)-(3): $M_{s} \simeq 1.267$, $u_{s} \simeq 435.677 \mathrm{~m} / \mathrm{s}$ and $u_{\text {air }} \simeq 136.881 \mathrm{~m} / \mathrm{s}$.

\section{ILES METHODOLOGY}

\section{A. Governing Equations}

Richtmyer-Meshkov interactions typically occur at very high Reynolds number. In this limit, we assume that $R e \rightarrow \infty$ and neglect the viscous terms in the

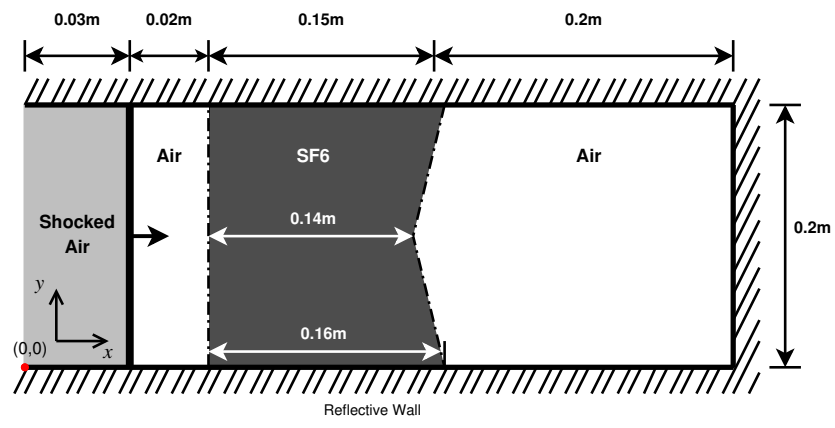

FIG. 2. Illustration of the inverse-chevron (IC) boundary and initial conditions.

TABLE II. Initial fluid properties for the inverse-chevron (IC) case at an initial Atwood number of $\left|A_{t}\right| \simeq 0.6853$.

\begin{tabular}{lccc}
\hline \hline & Shocked Air & Air & $\mathrm{SF}_{6}$ \\
\hline Density $\left(\mathrm{kg} / \mathrm{m}^{3}\right)$ & 1.7264 & 1.184 & 6.34 \\
Pressure $(\mathrm{Pa})$ & 170,600 & 100,000 & 100,000 \\
$\gamma$ & 1.4 & 1.4 & 1.076 \\
\hline \hline
\end{tabular}

Navier-Stokes equations. The dissipation present in the iLES is characteristic of the numerical scheme employed, i.e. is implicit. We have shown ${ }^{24}$ that for the numerical scheme and test-case pertinent herein, the effective Reynolds number is sufficiently high, i.e. $R e_{\text {eff }}>10^{5}$. The RANS simulations encompass dissipation through the dissipation term, $\varepsilon$. The dissipation is required for modelling the high-Re behavior of the turbulent mixing zones (TMZ).

The Euler equations employed here in the context of multi-component flows solve for the momentum and energy equations in conjunction with the (5-equation) quasi-conservative model of Allaire, Clerc, and Kokh ${ }^{29}$. The system of partial differential equations consists of two equations for the species densities:

$$
\begin{aligned}
& \frac{\partial z_{1} \rho_{1}}{\partial t}+\nabla \cdot\left(z_{1} \rho_{1} \mathbf{u}\right)=0 \\
& \frac{\partial z_{2} \rho_{2}}{\partial t}+\nabla \cdot\left(z_{2} \rho_{2} \mathbf{u}\right)=0
\end{aligned}
$$

momentum equation:

$$
\frac{\partial \rho \mathbf{u}}{\partial t}+\nabla \cdot(\rho \mathbf{u} \otimes \mathbf{u}+p \mathbf{I})=0
$$

the total energy equation:

$$
\frac{\partial \rho e_{t}}{\partial t}+\nabla \cdot\left[\left(\rho e_{t}+p\right) \mathbf{u}\right]=0
$$

and an additional equation for (one) volume fraction:

$$
\frac{\partial z_{1}}{\partial t}+\mathbf{u} \cdot \nabla z_{1}=0
$$


where $\rho, \mathbf{u}, e_{t}$, and $p$ are the densities, the velocity vector, the total specific energy, and the pressure, respectively. Furthermore, $\mathbf{I}$ is the identity tensor and $z_{1}$ is the volume fraction of air.

The above set of equations is used to model the initially sharp interfaces between the compressible fluids. As the flow develops, mixed cells form and the mixture model avoids spurious acoustic waves near the spread interface. To maintain the necessary numerical stability of the method, an isobaric closure postulating equal phase and mixture pressures is employed. Furthermore, both the fluids and mixture are assumed to share the same velocity (single-fluid model), and the addition of enthalpy diffusion terms ${ }^{30}$ associated with numerical diffusion can be neglected.

All fluids are modelled using an ideal gas equation of state given by $p=(\gamma-1) \rho i$ where $\gamma=c_{p} / c_{v}$ is the heat capacity ratio and $i=c_{v} T$ is the specific internal energy.

The single $\gamma$-formulation assuming the isobaric thermodynamic closure of the mixture is adopted here, according to which the mixture adiabatic index depends on the volume fractions of the individual components ${ }^{29}$ :

$$
\gamma=1+\frac{1}{\sum_{j} \frac{z_{j}}{\gamma_{j}-1}}
$$

where subscript $j$ stands for the individual fluids.

\section{B. iLES numerical methods}

We use iLES according to which specific features of the non-linear numerical method locally and dynamically produce similar effects to explicit sub-grid models used in conventional LES. All other assumptions are identical to the classical LES approach. We also assume that the resolved scales drive the turbulent mixing and the Schmidt number effects are negligible.

The numerical method solves the governing equations using a finite volume Godunov-type ${ }^{31}$ method. The intercell numerical fluxes are computed based on the solution to the Riemann problem using the reconstructed variables at the left and right of the cell interface. The iLES results presented here are from the same simulations conducted by Hahn et al. ${ }^{23}$ wherein further details can be found. In brief, the Harten, Lax, van Leer, and (the missing) Contact (HLLC) approximate Riemann solver of Toro, Spruce, and Speares ${ }^{32}$ is employed. A higher order of accuracy in smooth flow regions is achieved using Monotone Upstream-centered Schemes for Conservation Laws (MUSCL) extrapolation ${ }^{33}$ with the fifth-order limiter proposed by $\mathrm{Kim}$ and $\mathrm{Kim}^{34}$. The standard MUSCL extrapolation is additionally augmented using a low-Mach limiting scheme ${ }^{35}$, which involves an additional stage in the reconstruction process for the velocity vector. It ensures uniform dissipation of kinetic energy in the limit of zero Mach number $(M)$, extending the validity of the Godunov method for compressible solvers to at least $M \approx 10^{-4}$, via a progressive central differencing of the velocity components. The formulation of the underlying governing equations is not changed, and importantly the monotonicity of the density and scalar fields (e.g. volume-fraction) is maintained. Though the flow is mostly compressible, at a late time there exist large regions of the flow where mixing is occurring at a low-Mach number and thus require accurate modelling. Finally, for the discretization of the temporal derivatives, we use a third-order, three-stage, total variation diminishing Runge-Kutta (TVD-RK) ${ }^{36}$.

A structured Cartesian mesh comprising of $1280 \times 640 \times$ 320 cells in the streamwise, wall-normal and spanwise directions, respectively, is used for both of the considered test-cases, namely the double-planar (Fig. 1) and inversechevron (Fig. 2). The iLES data used herein have been thoroughly examined and analyzed previously ${ }^{24}$. Identical mesh size and numerical method were also employed by Hahn et al. ${ }^{23}$ when examining the effect of different interface perturbation spectra.

\section{Statistical quantities}

The flow variables, such as the velocity vector, $\mathbf{u}$, are decomposed into mean $\tilde{\phi}$ and fluctuating $\phi^{\prime \prime}$ components according to:

$$
\phi=\tilde{\phi}+\phi^{\prime \prime}
$$

where $\widetilde{(\cdot)}$ variables denote Favre, or mass, averaged variables, i.e. $\tilde{\phi}=\overline{\rho \phi} / \bar{\phi}$.

Additionally, $\overline{(\cdot)}$ variables represent Reynolds, or ensemble in space, averaged variables such that the fluctuation is defined by:

$$
\phi^{\prime}=\phi-\bar{\phi}
$$

The following relationships that relate the Favre and Reynolds averaged variables also hold: $\tilde{u}_{i}=\bar{u}_{i}+\overline{\rho^{\prime} u_{i}^{\prime}} / \bar{\rho}$, $u^{\prime \prime}=u_{i}^{\prime}-\overline{\rho^{\prime} u_{i}^{\prime}} / \bar{\rho}$, and $\overline{\rho u_{i}^{\prime \prime}}=0$.

The total mixing in the computational domain is defined according to:

$$
\mathrm{MIX}=\int \bar{\rho}^{2} \tilde{z}_{1} \tilde{z}_{2} d V
$$

where, for a binary mixture as considered here, the massfractions are related according to $\tilde{z}_{2}=1-\tilde{z}_{1}$. This integral quantity is not prone to statistical noise and is a simple way of measuring variations in the total amount of mixing in complex flows ${ }^{23}$.

The total turbulence kinetic energy is given by:

$$
\mathrm{TKE}=\int \bar{\rho} K d V
$$

where $K \equiv \tilde{k}=\left(\overline{\rho u^{2}}-\bar{\rho} \tilde{u}^{2}\right) / 2$, or otherwise:

$$
K=\frac{\overline{\frac{1}{2} \rho\left(u_{x}^{\prime \prime 2}+u_{y}^{\prime \prime 2}+u_{z}^{\prime \prime 2}\right)}}{\bar{\rho}}
$$


For the double-planar case the three-dimensional iLES results are averaged in the spanwise and wall-normal directions, $\tilde{f}(x)$, whereas for the inverse-chevron just in the spanwise direction, $\tilde{f}(x, y)$. Thus, for the $1 \mathrm{D}$ case the integral quantities are calculated along a line, i.e. $d V=L_{y} L_{z} d x$, whereas for the $2 \mathrm{D}$ case over a plane, i.e. $d V=L_{z} d A$. A direction is considered homogeneous in the sense that the mean flow gradients vanish and the statistical properties of the fluctuating field are invariant of translation along with it.

By investigating the above parameters, we aim to explain discrepancies between different models and help guide their further development.

\section{DESCRIPTION OF TURBULENCE MODELS}

\section{A. Youngs' multi-fluid model}

The turbulent mixing model is implemented for many fluids in a two-dimensional compressible Eulerian hydrocode $^{14}$. The calculations shown here are for mixing of two fluids. Hence, for simplicity, the model equations, which are based on the equations of two-phase flow with mass exchange terms, are given below for two-fluid mixing.

The volume fractions for the two "components" are denoted by $z_{r}(r=1,2)$. Initially, the two components correspond to the two initial fluids which have densities $\rho_{r}^{0}(r=1,2)$. As the fluids mix, mass is exchanged between the two components and this is used to represent the molecular mixing process ${ }^{21}$, i.e., the random mixture of the two fluids is represented by two components, one rich in initial fluid 1 and one rich in initial fluid 2 . Phase $r$ consists of fractions $\alpha_{r s}$ by volume of fluid $s$. Hence the density of component $r$ is $\rho_{r}=\alpha_{r 1} \rho_{1}^{0}+\alpha_{r 2} \rho_{2}^{0}$ and the mean density of the mixture is $\rho=z_{1} \rho_{1}+z_{2} \rho_{2}$. The model for 1D incompressible two-fluid mixing was described in detail by Kokkinakis et al. ${ }^{37}$. The extension to two- and three-dimensional compressible flow is summarized below.

The equations solved for the volume fractions $\alpha_{r s}$ are:

$$
\begin{array}{r}
\frac{\partial z_{r} \alpha_{r s}}{\partial t}+\bar{u}_{j} \frac{\partial z_{r} \alpha_{r s}}{\partial x_{j}}+\frac{\partial}{\partial x_{j}}\left[z_{r} \alpha_{r s}\left(\bar{u}_{r j}-\bar{u}_{j}\right)\right]= \\
\frac{\partial}{\partial x_{j}}\left(z_{r} D \frac{\partial \alpha_{r s}}{\partial x_{j}}\right)+\alpha_{r^{\prime} s} \Delta V_{r^{\prime} r}-\alpha_{r s} \Delta V_{r r^{\prime}}
\end{array}
$$

where $\bar{u}_{r j}, \bar{u}_{j}$ denote the volume-weighted mean velocities of component $r$ and of the mixture, respectively. Considering a binary mixture, here $r, s=1,2$, thus Eq. (15) covers all 4 possibilities. $\Delta V_{r s}$ is the rate of transfer of volume from component $r$ to component $s$ (component $r^{\prime}$ denotes the component which is not component $r$ ) and $D$ is the turbulent diffusivity. Summation over $s$ gives the equation for the volume fraction $z_{r}$. For the compressible case extra terms are included to allow for the differential compressibility of the two fluids and for pressure relaxation. Mass transport of component $r$ is given by:

$$
\frac{\partial\left(z_{r} \rho_{r}\right)}{\partial t}+\frac{\partial}{\partial x_{j}}\left(z_{r} \rho_{r} u_{r j}\right)=\Delta M_{r^{\prime} r}-\Delta M_{r r^{\prime}}
$$

where $\Delta M_{r s}=\rho_{r} \Delta V_{r s}$. Mass-weighted mean component velocities, $u_{r i}$, are needed for the momentum equations. Both $u_{r i}$ and $\bar{u}_{r i}$ include the effects of turbulent diffusion and their differences are attributed to within-component turbulent diffusion and are prescribed as described by Youngs $^{14}$ :

$$
u_{r j}-\bar{u}_{r j}=u_{r j}^{d}-\bar{u}_{r j}^{d}
$$

where

$$
u_{r j}^{d}=-\frac{D}{\rho_{r} z_{r}} \frac{\partial\left(\rho_{r} z_{r}\right)}{\partial x_{j}}
$$

and

$$
\bar{u}_{r j}^{d}=-\frac{D}{z_{r}} \frac{\partial z_{r}}{\partial x_{j}}
$$

Momentum equations are solved for mass-weighted mean component velocities, $u_{r i}$ :

$$
\frac{\partial\left(z_{r} \rho_{r} u_{r i}\right)}{\partial t}+\frac{\partial\left(z_{r} \rho_{r} u_{r i} u_{r j}\right)}{\partial x_{j}}=-z_{r} \frac{\partial p}{\partial x_{i}}+f_{r} \frac{\partial \mathrm{R}_{i j}}{\partial x_{j}}+X_{r r^{\prime} i}
$$

In Eq. (18), $p$ denotes the average pressure of the mixture, $f_{r}$ is the component $r$ mass fraction, $\mathrm{R}_{i j}$ is the Reynolds stress tensor and $X_{r r^{\prime} i}$ is the rate of transfer of momentum from component $r^{\prime}$ to component $r$ accounting for drag, added mass and mass exchange, as previously described ${ }^{37}$.

Since the flow is compressible, an equation for the internal energy of component $r$ is needed:

$$
\begin{array}{r}
\frac{\partial\left(z_{r} \rho_{r} i_{r}\right)}{\partial t}+\frac{\partial}{\partial x_{j}}\left(z_{r} \rho_{r} i_{r} u_{r j}\right)=-h_{r} p_{r} \frac{\partial \bar{u}_{j}}{\partial x_{j}} \\
+\frac{\partial}{\partial x_{j}}\left(z_{r} \rho_{r} D \frac{\partial i_{r}}{\partial x_{j}}\right)+z_{r} \varepsilon+i_{r^{\prime}} \Delta M_{r^{\prime} r}-i_{r} \Delta M_{r r^{\prime}}
\end{array}
$$

In Eq. (19), $p_{r}$ denotes the component $r$ pressure and the factor $h_{r}$ allows for the differential compressibility of component $r$, as defined by Youngs ${ }^{14}$.

Two further equations, for turbulence kinetic energy, $K$, and length scale, $L$, are used to close various terms in Eqs. (15) to (19): turbulent diffusion coefficients are $\sim \sqrt{K} L$, the drag term uses the length scale $L$, volume exchange rates are proportional to $\sqrt{K} L^{-1}$ and turbulence kinetic energy dissipation $\varepsilon$ is proportional to $\sim \rho K^{3 / 2} L^{-1}$. The additional equations are:

$$
\frac{\partial(\rho K)}{\partial t}+\frac{\partial\left(\rho K u_{j}\right)}{\partial x_{j}}=\frac{\partial}{\partial x_{j}}\left(\rho D_{K} \frac{\partial K}{\partial x_{j}}\right)+S_{K}-\varepsilon
$$


where $D_{K}$ is the turbulence kinetic energy turbulent diffusion coefficient and the source term $S_{K}$ is defined as:

$$
S_{K}=\left(u_{2 j}-u_{1 j}\right) X_{12 j}-\mathrm{R}_{i j} \mathrm{e}_{i j}
$$

where $\mathrm{e}_{i j}$ is the mean flow strain-rate defined as:

$$
\mathrm{e}_{i j}=\frac{1}{2}\left(\frac{\partial \tilde{u}_{i}}{\partial x_{j}}+\frac{\partial \tilde{u}_{j}}{\partial x_{i}}\right)
$$

The equation for the turbulence length scale $(L)$ for the two-fluid model is given as:

$$
\frac{\partial L}{\partial t}+u_{L j} \frac{\partial L}{\partial x_{j}}=\frac{\partial}{\partial x_{j}}\left(D_{L} \frac{\partial L}{\partial x_{j}}\right)+S_{L}+e_{L} L
$$

The source term, $S_{L}$, is proportional to the difference in the fluid velocities in the direction of mixing and $e_{L}$ denotes the strain rate in the direction of mixing ${ }^{14,21}$.

Several terms in Eqs. (15) to (23) require closure approximations. These are essentially the same as for the $1 \mathrm{D}$ incompressible case ${ }^{37}$ and the closure approximations require the selection of "model constants". A key test case used for mix model calibration is self-similar RT mixing for which the depth to which the lighter fluid penetrates the denser fluid is given by $h_{b}=\alpha A_{t} g t^{2}$, where $A_{t}$ is the Atwood number. The model constants are adopted from a previous study ${ }^{37}$ and are chosen to give $\alpha=0.06$, a typical experimental value.

\section{B. $K-L$ turbulence model}

The $K-L$ turbulent mixing model examined here was initially developed and proposed by Dimonte and Tipton ${ }^{19}$ for the turbulent self-similar regime of RayleighTaylor and Richtmyer-Meshkov instability (RMI) induced mixing. Here, a modified form of the model is employed, developed later by Kokkinakis et al. ${ }^{37}$. The modified $K-L$ model was previously altered and tested in the context of Rayleigh-Taylor instability (RTI) induced mixing $^{37}$, which is associated with gradual acceleration and is mostly incompressible. In contrast to the RTIinduced mixing considered previously ${ }^{37}$, this paper aims to assess further the performance of the mixing models in RMI-induced mixing cases, which involve impulsive-like acceleration and are highly compressible; at peak compression, the $\mathrm{SF}_{6}$ density is increased by about a factor of $\sim 3\left(\rho_{\max }^{\mathrm{SF}_{6}} \approx 20 \mathrm{~kg} / \mathrm{m}^{3}\right)$.

Similar to Kokkinakis et al. ${ }^{37}$, to reduce the numerical uncertainty, the $K-L$ model is implemented using the same numerical techniques as the iLES. In contrast to the iLES, however, the modified $K-L$ model is implemented using the fully-conservative multi-component formulation (4-equation model) of Allaire, Clerc, and Kokh ${ }^{29}$. Following the reconstruction process of ${ }^{37}$, spurious pressure oscillations that occur at the interface between variable $\gamma$ fluids are mitigated. The constants for the $20: 1$ density ratio $^{37}$ are implemented here and, similarly to the TF model, are calibrated to give $\alpha \sim 0.06$.
Pertinent to the discussion made later in this study, the turbulence kinetic energy source term $S_{K}$ is described next. According to Dimonte and Tipton ${ }^{19}$ :

$$
S_{K}= \begin{cases}C_{B} \bar{\rho} u_{\mathrm{t}}\left|A_{L j} g_{j}\right| & \text { if } \Theta<\Lambda_{\Theta} \Theta_{\mathrm{t}} \\ C_{B} \bar{\rho} u_{\mathrm{t}} \max \left(0, A_{L j} g_{j}\right) & \text { otherwise }\end{cases}
$$

where $\Lambda_{\Theta} \leqslant 1$ and $C_{B}$ are model coefficients. The criterion is based on the sudden acceleration of a fluid particle (estimated by the pressure gradient) and is not directly dependent on the CFL number. In Eq. (24), $g_{j}=-(1 / \bar{\rho}) \partial \bar{p} / \partial x_{j}$ is the acceleration, $A_{L j}$ is the local Atwood number while subscript $[j]$ denotes the considered Cartesian direction. The source term $S_{K}$ in Eq. (24) depends on the time scales of the mean flow, $\Theta$, and turbulent structures, $\Theta_{\mathrm{t}}$ defined as:

$$
\Theta=\bar{\rho} \sqrt{\left(\gamma \frac{\bar{p}}{\bar{\rho}}\right) /\left(\frac{\partial \bar{p}}{\partial x_{k}} \frac{\partial \bar{p}}{\partial x_{k}}\right)}, \quad \Theta_{\mathrm{t}}=\frac{L}{\sqrt{K}}
$$

he local Atwood number $\left(A_{L j}\right)$ used in the source term $S_{K}$ is calculated here according to Kokkinakis et al. ${ }^{37}$ :

$$
A_{L j}=\left(1-w_{L j}\right) A_{0 j}+w_{L j} A_{S S j}
$$

$A_{0 j}$ recovers the initial Atwood number at the interfaces between fluids where the density is discontinuous, whereas $A_{S S j}$ represents the local Atwood number in the self-similar regime where the density variation is gradual. The weighting factor $w_{L j}=\min \left(1, L / \Delta x_{j}\right)$ is the ratio of the turbulent mixing length $L$ to the grid cell size $\Delta x_{j}$. This ensures that when $L$ becomes sufficiently large, the Atwood number is solely based on $A_{S S j}$, while initially is dominated by $A_{0 j}$.

The initial Atwood number is calculated according to:

$$
A_{0 j}=\frac{\bar{\rho}_{i+1 / 2}-\bar{\rho}_{i-1 / 2}}{\bar{\rho}_{i+1 / 2}+\bar{\rho}_{i-1 / 2}}
$$

where $\bar{\rho}_{i \pm 1 / 2}$ are the mean left and right reconstructed values of the density at each opposite cell face in the $j$ direction of the $i t h$ cell, i.e.

$$
\bar{\rho}_{i \pm 1 / 2}=\left(\bar{\rho}_{i \pm 1 / 2}^{L}+\bar{\rho}_{i \pm 1 / 2}^{R}\right) / 2
$$

The self-similar cell Atwood number, $A_{S S j}$, is given by:

$$
A_{S S j}=C_{A} \frac{L}{\bar{\rho}+L\left|\frac{\partial \bar{\rho}}{\partial x_{j}}\right|} \frac{\partial \bar{\rho}}{\partial x_{j}}
$$

where $C_{A}$ is a model constant, and

$$
\frac{\partial \bar{\rho}}{\partial x_{j}}=\frac{\bar{\rho}_{i+1 / 2}-\bar{\rho}_{i-1 / 2}}{\Delta x_{j}}
$$

Further details and model constant coefficients are given in Kokkinakis et al. ${ }^{37}$. 


\section{Modifications}

In contrast to the previous study of Kokkinakis et al. ${ }^{37}$, the Reynolds stress tensor is modelled here using the full form of the Boussinesq eddy viscosity assumption:

$$
\mathrm{R}_{i j}=\frac{2}{3} \bar{\rho} K \delta_{i j}-2 \mu_{\mathrm{t}}\left(\mathrm{e}_{i j}-\frac{1}{3} \mathrm{e}_{k k} \delta_{i j}\right)
$$

where $\mu_{\mathrm{t}}$ is the turbulent viscosity and $\mathrm{e}_{i j}$ is the mean flow strain-rate given by Eq. (22).

Additionally, Dimonte and Tipton ${ }^{19}$ suggested that the local Mach number limits the Reynolds stress tensor $\left(\mathrm{R}_{i j}\right)$. Therefore, we linearly scale $\mathrm{R}_{i j}$, such that it approaches zero as the Mach number becomes one.

A posteriori analysis of iLES data indicates that a threshold in the production of $K$ is reached when the eddy size $L$ exceeds a critical value of the mixing width $W_{c}$ (at the first interface), so $L \geqslant W_{c}$. By post-processing the iLES data and comparing to the modified $K-L$ model results, it has been identified that the TKE begins to diverge when $W_{c} \approx 0.00625 \mathrm{~m}$. Above this threshold, the source term for turbulence kinetic energy production $\left(S_{K}\right)$ is reformulated to reduce according to:

$$
S_{K}^{L}=S_{K} \times \min \left[1,\left(\frac{W_{c}}{L}\right)^{2}\right]
$$

The $K$ - $L$ model of Kokkinakis et al. ${ }^{37}$ has been further enhanced by the following additions:

- the realizability conditions of Vreman, Geurts, and Kuerten ${ }^{38}$ are imposed on the Reynolds stress tensor, as detailed in Eq. $(28)^{39}$;

- the turbulent viscosity is limited according to the two-dimensional isotropic diffusion limiter, $S_{F}$, (see Eq. $(29)^{39}$ ), applicable here only to the twodimensional IC case;

- the time-step size considers the turbulent diffusion fluxes according to Eq. $(30)^{39}$;

\section{Turbulence model initial conditions}

The $2 \mathrm{D}$ interface surface $(y-z$ plane) in the 3D iLES had perturbations superimposed to simulate the effect of the wire mesh present in the experiment ${ }^{22}$, used to keep the two gases separated until they were shocked. A practical method of representing this two-dimensional interface is the summation of several individual wave modes following an assumed surface perturbation power spectrum as proposed by Youngs ${ }^{40}$.

The RANS simulations are initialized at the same time as the $3 \mathrm{D}$ simulation. Time $t=0 \mathrm{~ms}$ is defined as the moment the shock wave reaches the first interface. Based on the shock wave velocity, given in $\S I I$, it is possible to estimate the time it takes for the shock wave to reach the first interface. Hence the initial condition is given at $t \approx-0.041 \mathrm{~ms}$.

The initial mean flowfield variables in the RANS simulation are obtained by averaging the 3D iLES initial condition in the homogeneous directions onto an equivalent resolution mesh. To get the initial conditions for the coarser RANS grids, the averaged fine solution is further (2D area-, or 1D length-) averaged onto the coarser grids. For the inverse-chevron case, in particular, this helps avert the generation of artificial instabilities resulting from the significant "step-like"discretization of the mass-fraction at the inclined interface on coarser grids. The volume-averaging effectively leads to a diffuse material interface on the coarser grids.

Finally, initial values for the additional turbulence model variables, in this case, the turbulence kinetic energy $(K)$ and turbulence length scale $(L)$, need to be specified. Several steps described next will obtain these.

The procedure begins with the $3 \mathrm{D}$ iLES results obtained at a time just after the first planar interface has been shocked $(t=0.5 \mathrm{~ms})$. The above allows adequate time for the bubbles and spikes generated by the RM instability to form and their respective lengths $h_{b}$ and $h_{s}$ to be easily estimated. The procedure is briefly summarized as follows:

1. Estimate mean location of interface, bubbles and spikes just after the first interface is shocked $(t=0.5 \mathrm{~ms})$ using the Favre-averaged iLES massfraction;

2. Obtain bubble $\left(h_{b 5}\right)$ and spike amplitudes $\left(h_{s 5}\right)$ at same post-shock time $(t=0.5 \mathrm{~ms})$;

3. Calculate the velocity of the amplitudes according to $V_{x 5}=d h_{x 5} / d t$, where subscript $x$ denotes either $b$ for bubble or $s$ for spike;

4. Estimate velocity of amplitudes at $t=0$ (just after shock) based on the drag-buoyancy model ${ }^{41}$;

5. Estimated velocity amplitudes $V_{i 0}$ are then used to obtain an estimate for the turbulence kinetic energy $K_{i}=0.5 u_{\mathrm{t}}^{2}$

6. $L$ is assumed to be equal to the initial $(t=0)$ perturbations width at the interface used in the $3 \mathrm{D}$ iLES.

Note that the obtained values for the planar interface are also subsequently used for the inclined surface as well.

By post-processing the 3D high-resolution iLES results of Hahn et al. ${ }^{23}$ at $t=0.5 \mathrm{~ms}$, it is possible to estimate the bubble, spike and interface location. Initially, the $3 \mathrm{D}$ results are averaged onto a $2 \mathrm{D}$ plane from which the required positions are extracted. Moving along the $x$ direction, the position of the spike $\left(X_{s}^{t}\right)$ is calculated as the location at which the volume fraction $(\tilde{z})$ first becomes less than or equal to $99 \%$, the interface location $\left(X_{i}^{t}\right)$ where $\tilde{z}$ is less than or equal to $50 \%$, and the bubble location $\left(X_{b}^{t}\right)$ where $\tilde{z}$ first becomes greater than or 
TABLE III. Post-processed average amplitude positions at $t=0.5 \mathrm{~ms}$ for the $k^{-2}$-spectrum initial interface perturbation.

\begin{tabular}{lcc}
\hline \hline$X_{\text {int } 5}(\mathrm{~m})$ & $X_{b 5}(\mathrm{~m})$ & $X_{s 5}(\mathrm{~m})$ \\
\hline 0.0935317 & 0.095514 & 0.0913061 \\
\hline \hline
\end{tabular}

TABLE IV. Initial $K$ values for $k^{-2}$-spectrum just after shock.

\begin{tabular}{cc}
\hline \hline$K_{b 0}\left(\mathrm{~m}^{2} / \mathrm{s}^{2}\right)$ & $K_{s 0}\left(\mathrm{~m}^{2} / \mathrm{s}^{2}\right)$ \\
\hline 44.84 & 24.25 \\
\hline
\end{tabular}

equal to $1 \%$. The process is repeated until the full height of the domain ( $y$-direction) is covered, and the required averages are obtained. Table III shows the values obtained for the average position of the interface, bubble and spike by post-processing the Favre-averaged volume fraction $(\tilde{z})$ iLES results ${ }^{23}$ at $t=0.5 \mathrm{~ms}$.

The amplitude widths $h_{5 i}$ are then calculated as $h_{b 5} \simeq$ $1.9823 \mathrm{~mm}$ and $h_{s 5} \simeq 2.2256 \mathrm{~mm}$ for the bubble and spike, respectively.

Before shock, the planar interface is located at $X_{\text {int } 0}=$ $0.05 \mathrm{~m}$. Although there are no distinctive bubble and spike features formed yet, it is assumed that they are equal to the spectral amplitude perturbation given to the volume fraction, which in either case is equal to twice the cell width. Therefore the initial bubble and spike amplitudes are both assumed to be $h_{i 0}=0.625 \mathrm{~mm}$.

A value for the bubble and spike velocities at $t=0.5 \mathrm{~ms}$ can now be obtained using the relation $V_{i}=\partial h_{i} / \partial t$. The calculated bubble and spike velocities at $t=0.5 \mathrm{~ms}$ are $V_{b 5}=2.7146 \mathrm{~m} / \mathrm{s}$ and $V_{s 5}=3.2012 \mathrm{~m} / \mathrm{s}$, respectively.

If the shock is considered to impart an impulse to the bubbles and spikes at the interface, their ensuing motion can be described by equating inertia with drag. The postshock values of the amplitudes velocities are computed based on Youngs' coupled bubble and spike buoyancy drag model ${ }^{41}$, which has been shown to give satisfactory results $^{19}$ :

$$
\begin{aligned}
& V_{b 0}=V_{b 5}+C \frac{V_{b 5}\left|V_{b 5}\right|}{h_{2}} d t \\
& V_{s 0}=V_{s 5}+\frac{C}{\sqrt{R}} \frac{V_{s 5}\left|V_{s 5}\right|}{h_{2}} d t
\end{aligned}
$$

where $h_{2}=\min \left(h_{b 5}, h_{s 5}\right)=0.0019823 \mathrm{~m}, R=\rho_{2}^{*} / \rho_{1}^{*} \simeq$ 6.234 where superscript [*] denotes post-shock conditions, $d t=0.5 \mathrm{~ms}$ and $C=3.667^{41}$. This results in a bubble and spike velocity of $V_{b 0} \simeq 9.4702 \mathrm{~m} / \mathrm{s}$ and $V_{s 0} \simeq 6.9640 \mathrm{~m} / \mathrm{s}$, respectively.

Assuming that the calculated amplitude velocity $V_{i}$ is equivalent to the turbulent velocity $u_{\mathrm{t}}$, it is possible to obtain initial values for $K$ based on $K=u_{\mathrm{t}}^{2} / 2$, shown in Table IV.

The $K-L$ parameters are initialized at the interface located at a time-step just after the shock. The same values are assumed to hold for the second interface too.
Turbulence models have been shown ${ }^{18,42}$ to be sensitive to the prescribed initial condition values, i.e. $K_{0}$ and $L_{0}$. Similar to Grinstein ${ }^{43}$, a sequential LES / (unsteady) RANS hybrid simulation strategy is essentially adopted here in which iLES generated unsteady data provides physics based initial conditions (IC) to the turbulence models; for $K_{0}$ via Youngs' coupled bubble and spike buoyancy drag model ${ }^{41}$. The RANS results obtained are deemed satisfactory and we do not carry out a parametric study of the models' sensitivity on IC.

\section{RESULTS}

The accuracy of the $K-L$ and TF models is investigated using data obtained from high-resolution iLES for the double-planar (DP) and inverse-chevron (IC) testcases ( $\S \mathrm{II})$. The DP case is used as a preliminary assessment of the mixing models performance in accurately predicting the formation and evolution of simple planar RMI-induced mixing. The (spatially) one-dimensional formulation does not require the cross-terms. For the IC case, we use the two-dimensional formulation of the models. Importantly, this will permit the weaknesses of each model in one-dimension to be separated from those that are pertinent to two-dimensional flows.

The primary rationale behind using a turbulence model to simulate turbulent mixing is to obtain, in a computationally inexpensive manner, as accurate as possible an estimate of the volume fraction profile and mixing zone width. A turbulent viscosity $\left(\mu_{\mathrm{t}}\right)$ is assumed that acts to diffuse the mixing interface, mimicking the effect of turbulence. In the case of most multi-component twoequation turbulence models, such as the $K-L$ and TF models considered here, the turbulent viscosity is a function of the modelled turbulence kinetic energy $(K)$ and turbulence length scale $(L)$ or dissipation rate $(\varepsilon)$.

Thus this study considers only those comparisons that are commonly of more importance and practical interest. These include integral quantities such as the total-mix, MIX in Eq. (12), and total- $K$, TKE in Eq. (13), as well as the spatial profiles of the volume fraction $(\tilde{z})$ and turbulence kinetic energy $(K)$, during the entire evolution of the interfaces. For both cases considered here, the growth of the mixing layers is not only dictated by the passage of a single shock, or reshock but multiple subsequent reshocks of varying degrees of strength and Atwood number sign. The passage of expansion fans generated when a (re-)shock wave exits the heavier $\mathrm{SF}_{6}$ further influences the evolution of the mixing layers. Thus the performance of the models is assessed here under conditions encountered more frequently in more complex engineering applications.

Figure 3 is an $x-t$ diagram generated for the considered double-planar test-case clearly illustrating the complexity of the developing RMI and ensuing turbulent mixing zones (TMZ). During the course of the simulation, a multiple number and type of pressure waves form and 


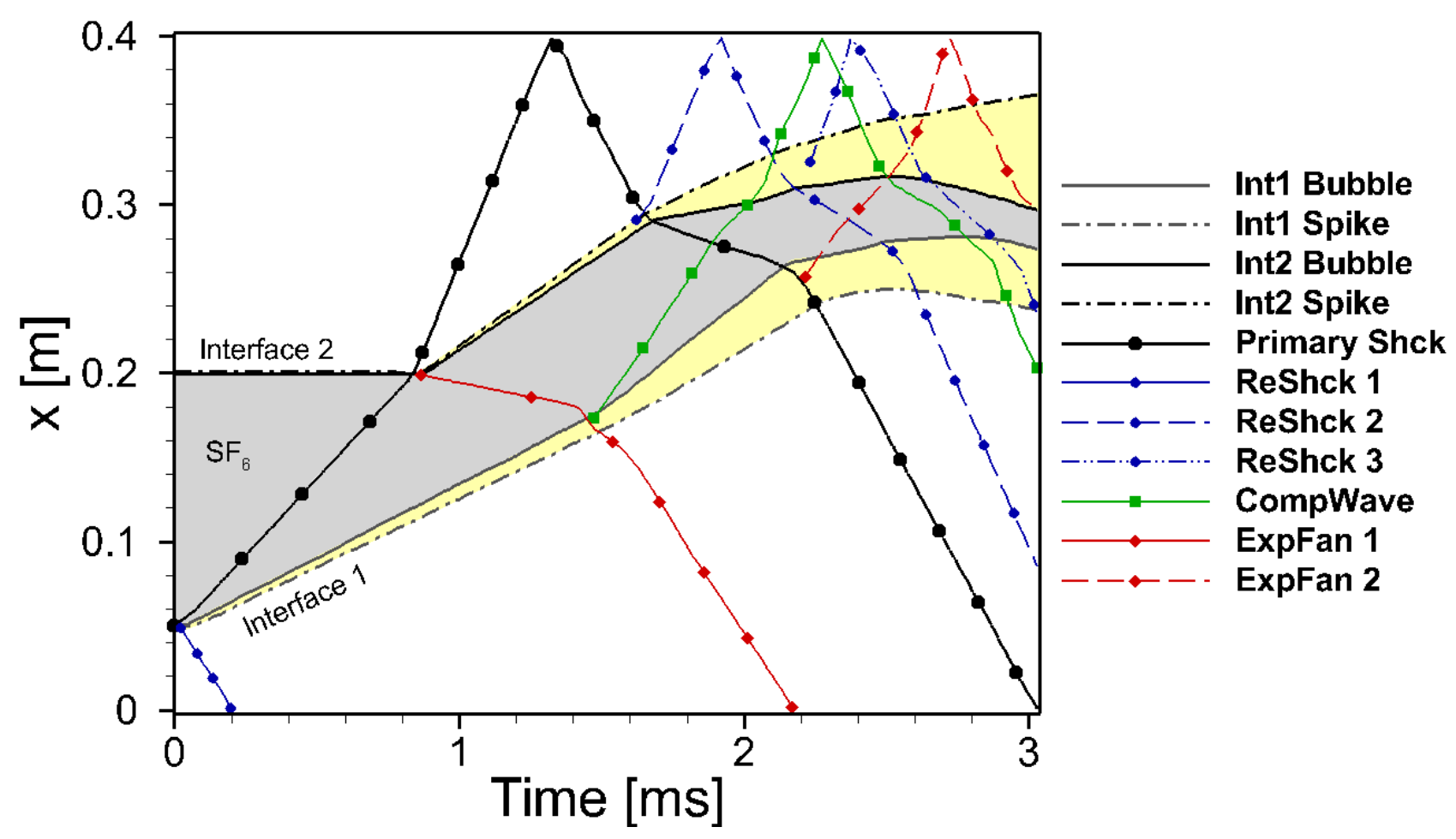

FIG. 3. An $x-t$ diagram of the considered double-planar case.

interact with the developing TMZ.

The $K-L$ model ( $\S I V B$ ) is henceforth labeled as KL, whereas when implemented with all the modifications described in $\S I V B 1$ as $\mathrm{KL}_{\mathrm{M}}$. Finally, TF abbreviates the two-fluid model discussed in §IV A.

\section{A. Double planar case (1D)}

We performed the calculations on a one-dimensional grid of 100 cells size (streamwise direction). The totalmix and total- $K$ are shown in Figures 4 and 5.

Until $t=1.60 \mathrm{~ms}$, the total-mix appears to be slightly over-predicted by the $K-L$ model variants. However, thereafter and until $t \approx 3.0 \mathrm{~ms}$, the $K-L$ models are closer to iLES. The $\mathrm{KL}_{\mathrm{M}}$ variant performs best. The overprediction before $t=1.6 \mathrm{~ms}$ is a result of the higher numerical dissipation of the Eulerian Finite-Volume framework within which the $K-L$ model has been implemented. The semi-Lagrangian framework used for the two-fluid model, for the same grid resolution, is less dissipative. The under-prediction of the total-mix, MIX, at $t \approx 2.0 \mathrm{~ms}$, substantiated by the volume fraction profiles of the fluid interfaces at $t=1.90 \mathrm{~ms}$ (Fig. 6(a)), is caused by the insufficient production of turbulence kinetic energy, $K . K$ is produced during the transit of the expansion wave at the left interface, and the reshock at the right interface, at $t \approx 1.65 \mathrm{~ms}$ (Fig. 6(b)).

Overall, the TF model gives the best agreement to the iLES up until $t \approx 2.70 \mathrm{~ms}$. The Lagrangian phase of the numerical scheme introduces little to no numerical dissipation for such a flow until the remap phase is required. Nonetheless, the rate of increase of MIX for the TF model, evident by its slope in Fig. 4, closely matches that of the iLES data. After $t \approx 2.70 \mathrm{~ms}$, the magnitude mismatch between the TF and iLES total-mix is primarily attributed to the insufficient production of turbulence kinetic energy during the weaker late-time reshocks of the mixing layer, as evident in Fig. 8(b).

Fig. 5 suggests that the models manage to reasonably predict the behaviour of the TKE. In the time frame, $t \approx 1.4-1.6 \mathrm{~ms}$, during which an expansion fan exits the $\mathrm{SF}_{6}$ from the first (left) interface, all models give the correct behaviour for the turbulence kinetic energy $(K)$ production.

Shortly after that, at $t=1.6 \mathrm{~ms}$, the second (right) interface is reshocked by the incident shock, which was earlier reflected at the right solid boundary. During this first of the many reshock events to follow, the TF model manages to accurately predict the production of $K$, in contrast to either of the $K-L$ model variants examined; of the latter, the $\mathrm{KL}_{\mathrm{M}}$ option performs better. The magnitude of the turbulence kinetic energy $(K)$ at either interface appears to be inconsistent with that of the iLES (Fig. 6(b)). The lower $K$ at the left interface is attributed to rapid dissipation following the transit of the expansion fan. Thus the correct amount of turbulence kinetic energy dissipation, $\varepsilon$, during and in particular following an interface shock is as an important parameter as the production of turbulence kinetic energy, $S_{K}$. 


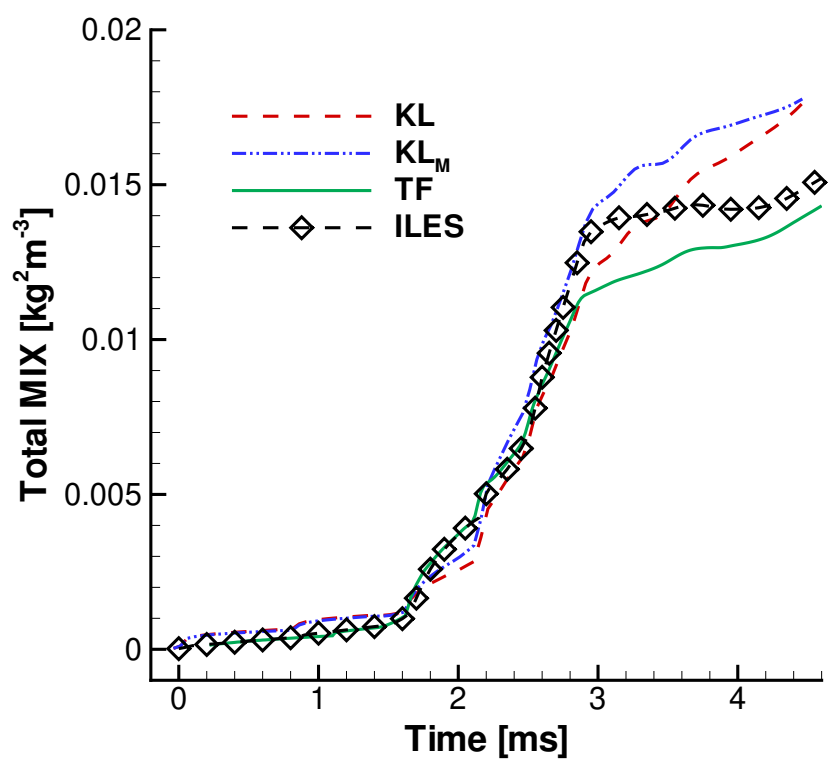

FIG. 4. Total-mix (MIX) versus time for the double-planar case.

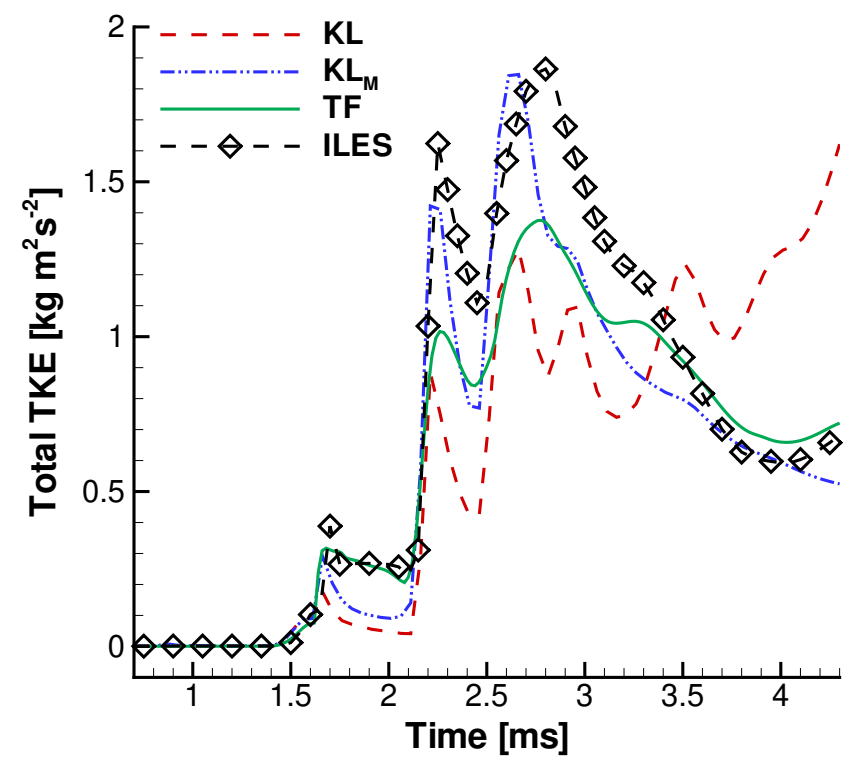

FIG. 5. Total-K (TKE) versus time for the double-planar case.

The almost simultaneous reshock of both interfaces that takes place at $t \approx 2.22 \mathrm{~ms}$ (see Fig. 3 ), brings the magnitude of the TKE predicted by the $K-L$ model back inline with the iLES. The $\mathrm{KL}_{\mathrm{M}}$ variant is once more in better agreement to the iLES and is attributed to the realizability conditions imposed on the Reynolds stress tensor. The TF model performs admirably, as both the volume fraction and turbulence kinetic energy profiles are in excellent agreement with the iLES at both $t=1.90$ and $2.22 \mathrm{~ms}$ time instances, as evident in Figs. 6 and 7 respectively.

Though up until $t \approx 2.70 \mathrm{~ms}$ the TF model has followed the iLES TKE precisely, the production of $K$ henceforth begins to be under-predicted. Examining Fig. 8(b) reveals that the turbulence kinetic energy across the first interface is under-predicted, while both of the $K$ - $L$ model variants give a much closer estimate to the iLES. However, the better agreement of the $K-L$ model at the left interface is coincidental. The width of the first interface for the $K-L$ model variants is much narrower during the previous time (compare $\tilde{z}$ profiles at $t=2.22 \mathrm{~ms}$ in Fig. 7(a) for example). Consequently, the density gradient on which the turbulence kinetic energy production source term $\left(S_{K}\right)$ depends on is much larger, resulting in the observed excess TKE production at $t \approx 2.70 \mathrm{~ms}$.

The late time, i.e. $t \geq 2.70 \mathrm{~ms}$ considered here, poses several problems to the turbulence models accuracy:

1. earlier inaccuracies accumulate;

2. the mixing regions become subsonic, and thus numerical dissipation increases for compressible flow solvers; and

3. length scale saturation can occur once $L \approx 0.1 L_{z}$ as demonstrated by Thornber ${ }^{44}$.

The KL model exhibits unusual oscillations at the late time (after $t=2.70 \mathrm{~ms}$ ), which become significantly reduced for the $\mathrm{KL}_{\mathrm{M}}$ variant, as a result of the use of Eq. (30). The oscillations are produced by an overestimation of the turbulent production source term, $S_{K}$, in the $K-L$ model and are found to occur only at the left interface at late time. The molecular mixedness between the two components results in an over-estimation of $K$ production across the mixing width of the left interface.

We improve the model accuracy by modifying the turbulence kinetic energy source term according to Eq. (30). The modification is ad-hoc and requires a-posteriori knowledge of a mixing width $\left(W_{c}\right)$ above which the production of $K$ is considered excessive. More complex turbulence models, such as the BHR model, introduce a transport equation for the density-specific-volume covariance ( $b$-parameter). The density-specific-volume covariance $b$ is a measure of how well the instantaneous density and specific volume properties of the fluid agree. It can be shown that $b=\overline{\rho^{\prime 2} / \bar{\rho} \rho^{45,46}}$. In mixed regions where $b=0$, the fluids are considered perfectly mixed. For completely segregated materials, e.g. the immiscible case and binary systems, $b$ has a simple formula: $b=z_{1} z_{2}\left(\rho_{1}-\rho_{2}\right)^{2} / \rho_{1} \rho_{2}{ }^{46}$, where $z_{1}$ and $z_{2}$ are the volume fractions associated with the different fluid components in a binary mixture. As a result, the initial premixed value of $b$ increases to infinity as the initial density ratio in a binary system increases.

It is not presently possible to establish a metric for the mixedness using the existing $K-L$ model formulation. Instead, an ad-hoc ratio of the turbulence length scale $L$ to the grid size $\Delta x$ is taken, based on the assumption that when the turbulence length scale becomes significantly large relative to the cell size, the fluids are homogeneously mixed. Besides, as $L$ increases relative to the 


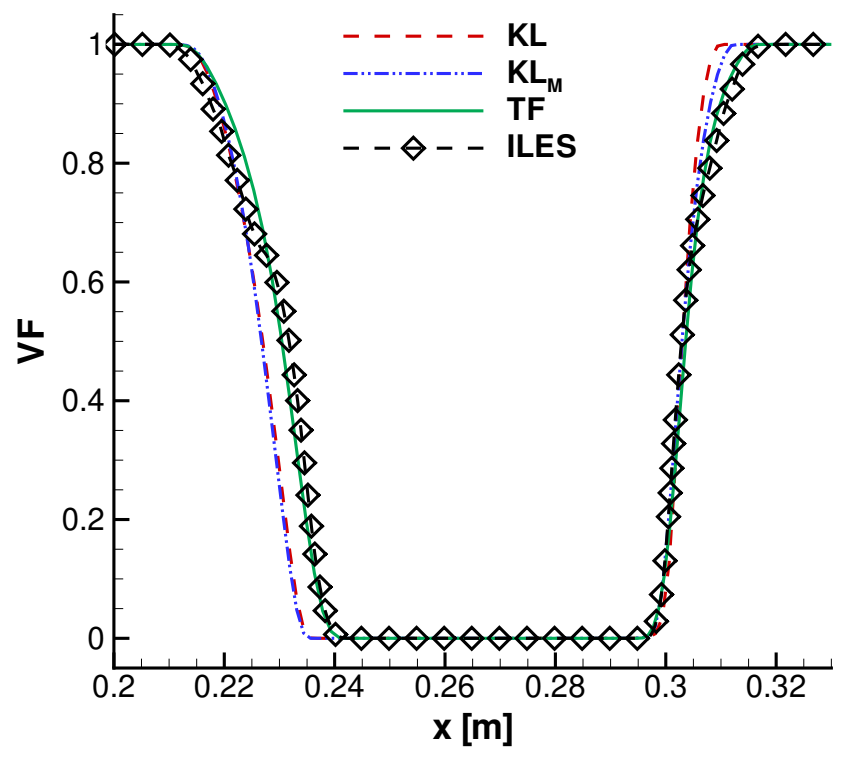

(a)

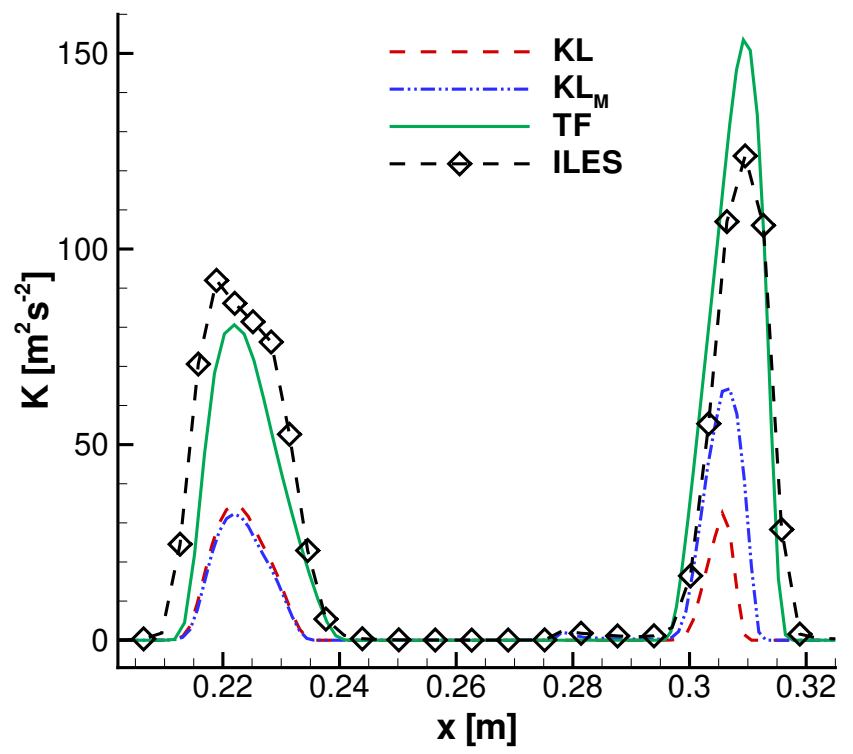

(b)

FIG. 6. Plots of the (a) volume fraction, $\tilde{z}$, and (b) turbulence kinetic energy, $K$, profiles at $t=1.90 \mathrm{~ms}$ for the double-planar case.

smallest domain size, length scale saturation can occur as $L \approx 0.1 L_{z}{ }^{44}$, further hindering the continuous steady growth rate of the interface. Nonetheless, we found that the above assumption cannot distinguish with sufficient accuracy such regions and consequently the turbulence kinetic energy production $\left(S_{K}\right)$ at the right interface is unnecessarily reduced as Fig. 9(b) reveals. The overestimation of $K$ also results in an excessive diffuseness of the left interface (see Fig. 9(a)). This, in turn, influences the total-mix (MIX) estimation for the KL model variant. As evident in Fig. 5, the TKE begins to skyrocket at a late time due to the over-estimation of $S_{K}$ at the

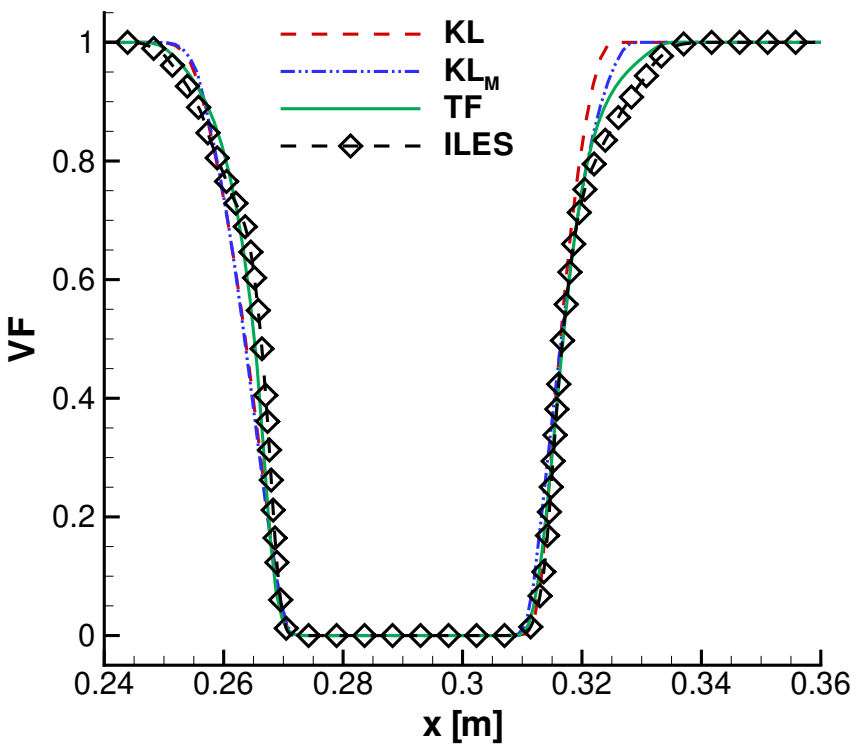

(a)

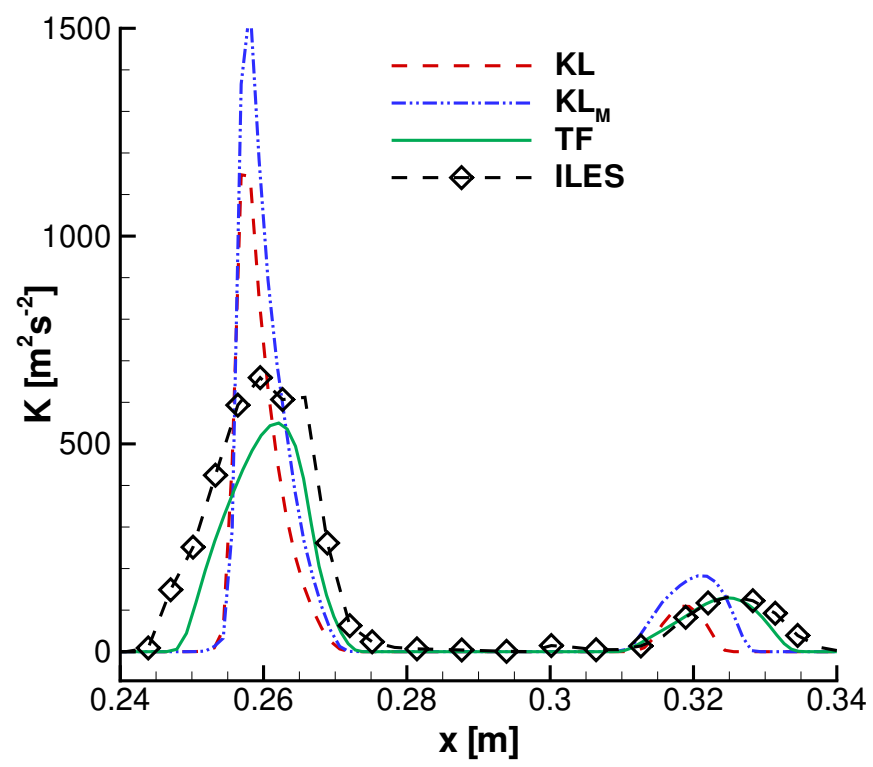

(b)

FIG. 7. Plots of the (a) volume fraction, $\tilde{z}$, and (b) turbulence kinetic energy, $K$, profiles at $t=2.22 \mathrm{~ms}$ for the double-planar case.

left interface (Fig. 9(b)). Consequently, it causes the left interface to become excessively diffuse (Fig. 9(a)), subsequently leading to a sharp rise in MIX as well (Fig. 4).

Another challenge for the turbulence models is the change in the dynamics of the spike, and bubble RM features driving their self-similar growth. The reshock at the left interface $(t \approx 2.70 \mathrm{~ms})$ causes a reversal of its direction and the rapid collapse of the initially formed bubbles and spikes. The resulting highly mixed state at the left interface significantly alters its growth rate and nullifies the initial model calibration. It is not a coincidence that the results obtained from the $K-L$ model at 


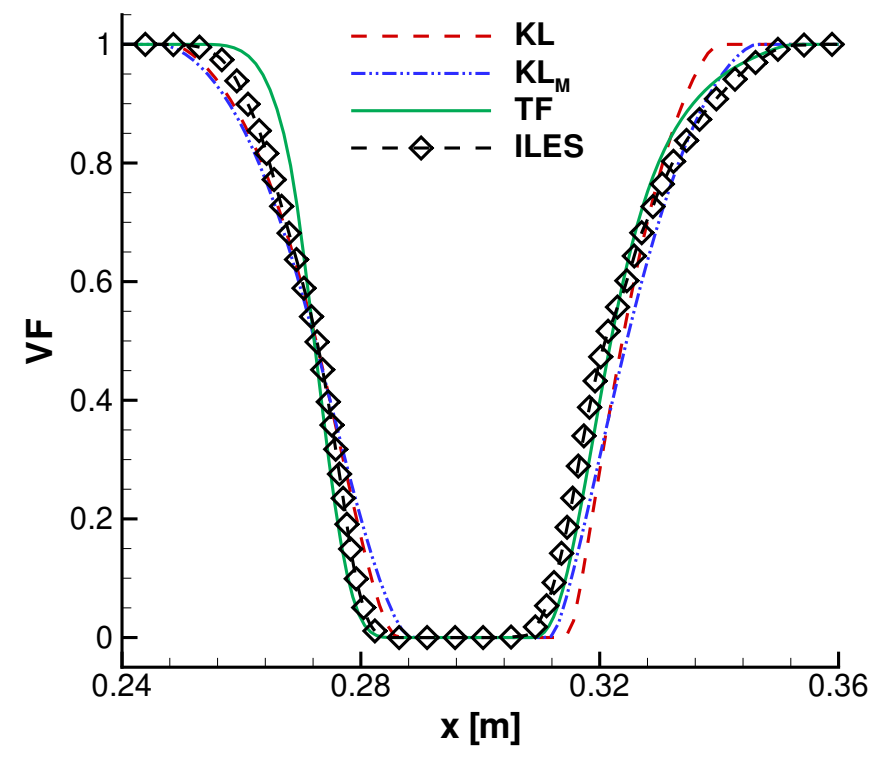

(a)

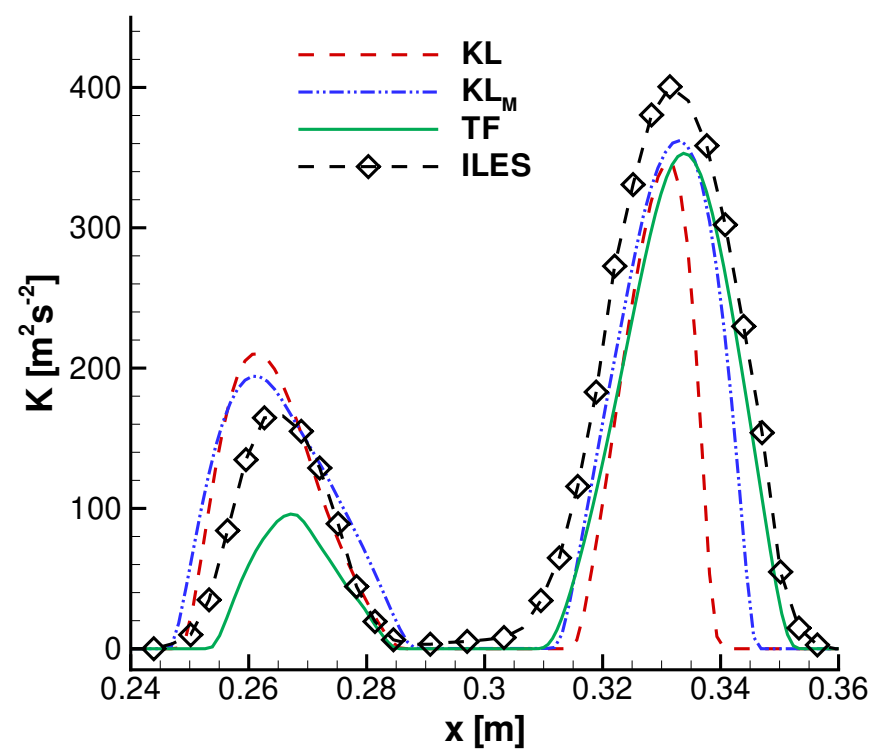

(b)

FIG. 8. Plots of the (a) volume fraction, $\tilde{z}$, and (b) turbulence kinetic energy, $K$, profiles at $t=2.70 \mathrm{~ms}$ for the double-planar case.

the right interface remain within reasonable agreement to iLES even at late time $t=3.82 \mathrm{~ms}$ (see Fig. 9). The above occurs because the shock does not experience a similar reversal of direction and subsequent the width collapse of the turbulent mixing zone (TMZ).

Overall, the TF model gives the most accurate results for both the $\tilde{z}$ and $K$ profiles even at a late time. The $\mathrm{KL}_{\mathrm{M}}$ model variant gives overall a satisfactory improvement over the KL variant. After the passage of the initial incident shock, the Atwood number describing the mixing interfaces increases as a result of the higher compression of the $\mathrm{SF}_{6}$. The $K-L$ model could be further improved by

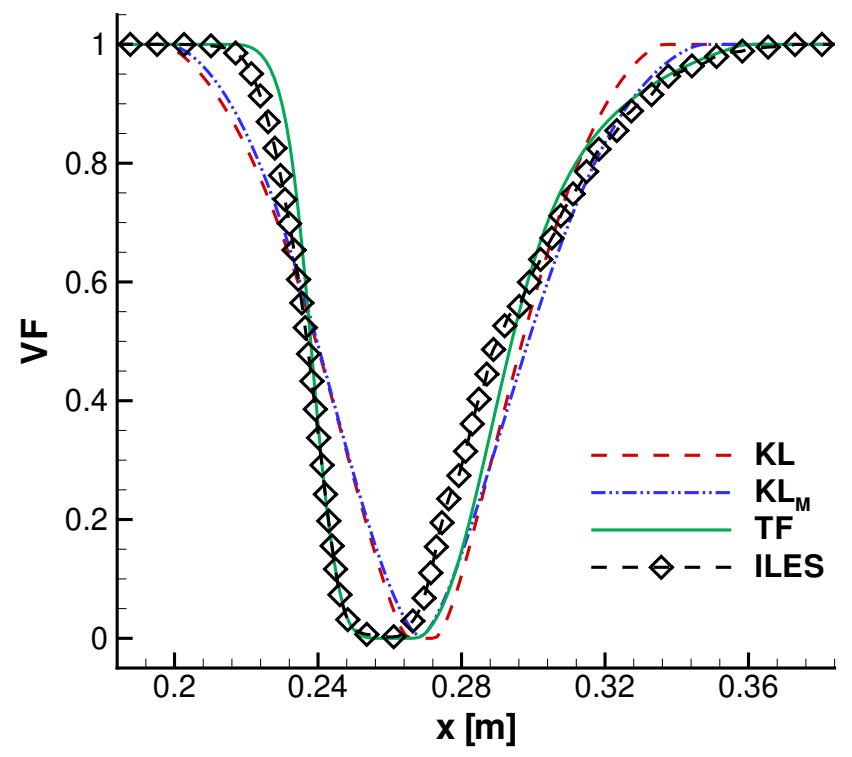

(a)

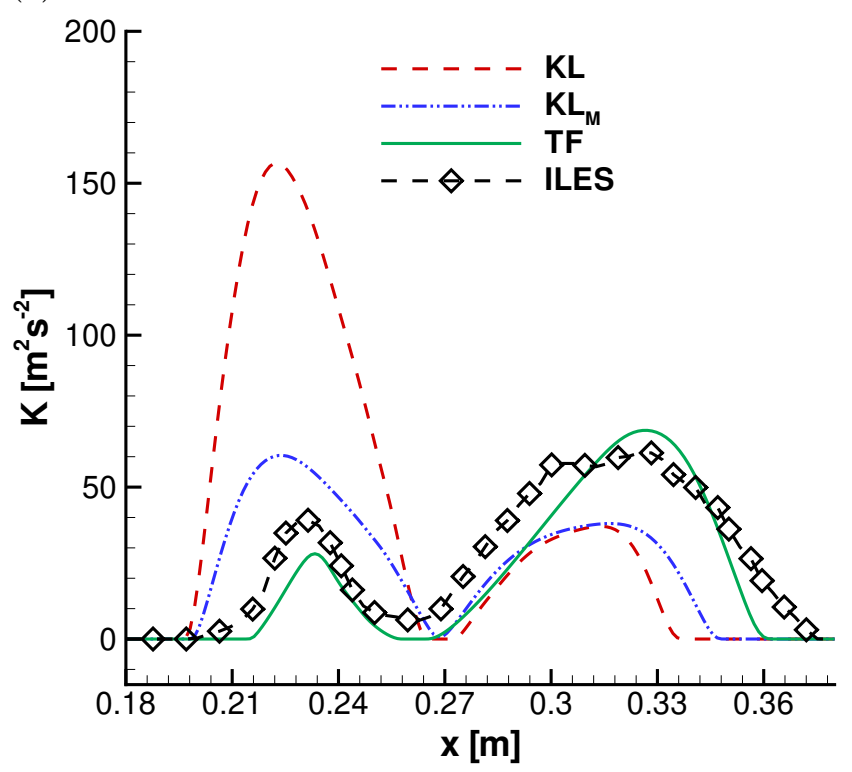

(b)

FIG. 9. Plots of the (a) volume fraction, $\tilde{z}$, and (b) turbulence kinetic energy, $K$, profiles at $t=3.82 \mathrm{~ms}$ for the double-planar case.

using constants calibrated for an Atwood number that, on average, is more representative of the double-planar case considered here, i.e. $A_{t} \approx 0.75$ (instead of $\approx 0.9$ ).

\section{B. Inverse Chevron (2D)}

Since the spanwise $(z$-)direction contains fluxes associated only with the turbulent field and that there is no mean flow gradient, the turbulence statistics are independent of translation, thus statistically homogeneous in the $z$-direction. Therefore, we model the 3D turbulent 


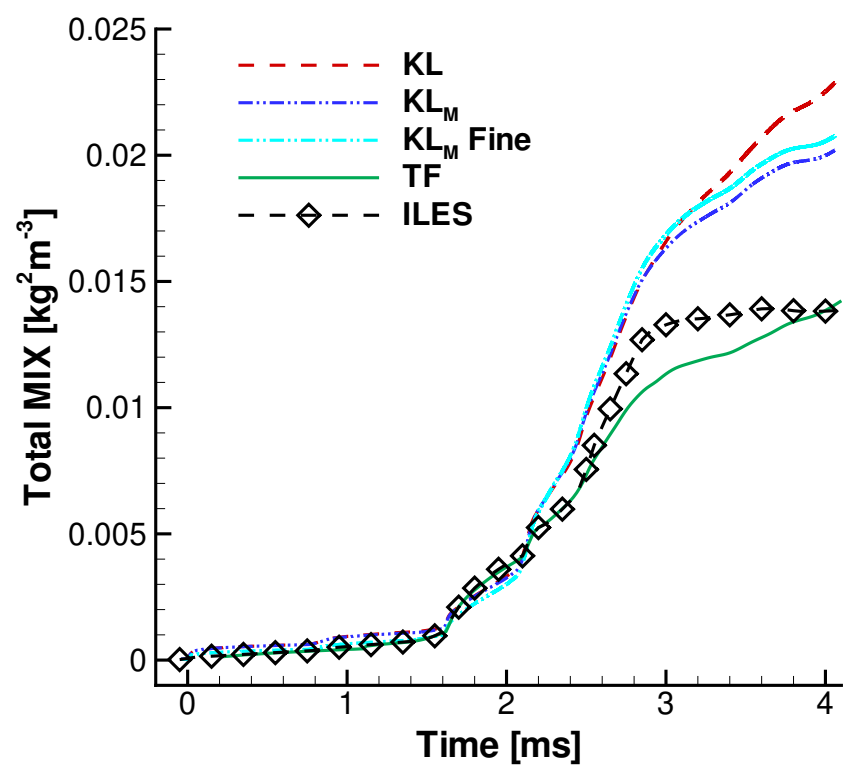

FIG. 10. Total-mix (MIX) versus time for the inverse-chevron case.

mixing in 2D ( $x$-y plane) using the TF and $K$ - $L$ models of $\S I V A$ and $\S I V B$.

For comparison and analysis purposes, the highresolution inverse-chevron iLES results of Hahn et al. ${ }^{23}$ are used. These include integral parameters such as the total-mix and total- $K$ variation in time (see $\S I I I C$ ), as well as $\tilde{z}$ and $K$ contour plots at two late time instances, namely 2.70 and $3.30 \mathrm{~ms}$. To make the comparison compatible, the MIX and TKE integral quantities provided in Hahn et al. ${ }^{23}$ have been re-calculated here using the spanwise averaged iLES flowfield. We performed calculations on a Cartesian mesh $320 \times 160(x-y)$ cells. For grid convergence of the $K-L$ turbulence model, a finer grid containing $640 \times 320$ cells is also considered (labeled as "Fine" in Figs. 10-15).

The inverse-chevron is a more demanding test-case since it involves not only the accurate modelling of RMIinduced mixing but also Kelvin-Helmholtz instabilities (KHI), which develop at the inclined interface. Moreover, it introduces several other challenges associated with the inclusion of cross-terms due to the additional spatial dimension. Both RM and KH types of instabilities form at the inclined interface post-shock, the former (RM) due to the impulsive acceleration, while the latter $(\mathrm{KH})$ due to the shearing resulting from the initial inclination. In the 3D iLES ${ }^{23}$, the first (left) planar interface forms RMIinduced mixing strictly. In contrast, the inclined inversechevron interface also developed KH-type instabilities as a result of the shear. Thus it is anticipated that the left interface will be susceptible to the same problems encountered previously for the double-planar case.

The total-mix (MIX) during early time $(t<1.6 \mathrm{~ms})$ is in close agreement to the iLES. Thereafter, and most notably post $\sim 2.7 \mathrm{~ms}$, the TF model follows the iLES

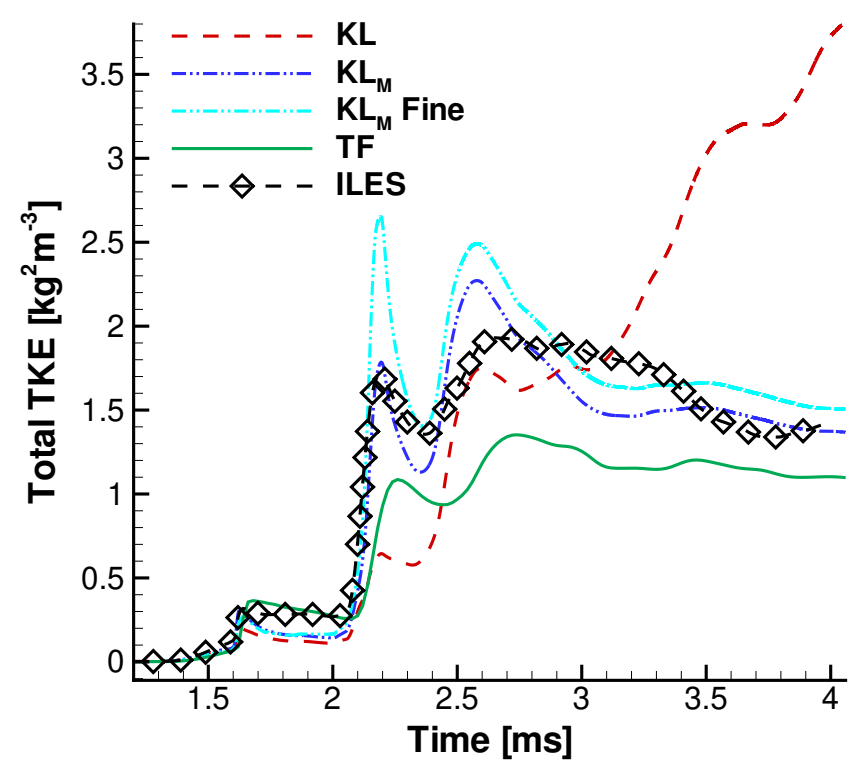

FIG. 11. Total-K (TKE) versus time for the inverse-chevron case.

MIX result more accurately while all $K-L$ model variants examined generally over-predict it (discussed later), as Fig. 10 shows. The sharper resolved interfaces on the fine mesh lead to a greater increase in the TKE during the reshocks at $t \approx 2.2 \mathrm{~ms}$ (Fig. 11). The initially large $60 \%$ overshoot in TKE rapidly reduces to under $10 \%$. However, the discrepancy then persists for the remainder of the simulation. Hence, though the $\mathrm{KL}_{\mathrm{M}}$ model variant is mostly insensitive to the grid resolution, some measurable differences can be discerned in integral properties such as the total-mix (MIX) and total- $K$ (TKE) as a result of the different, but prevalent, numerical diffusion at the early time.

With regards to the TF model, its TKE accurately follows that of the iLES up until $t=2.0 \mathrm{~ms}$ as Fig. 11 depicts. After that, the magnitude of the TKE is captured more accurately by $\mathrm{KL}_{\mathrm{M}}$. The TF model is also close to iLES. The TF model manages to resolve the early time total-mix (MIX) exceptionally well as Fig. 10 demonstrates. After $t \approx 2.6 \mathrm{~ms}$, there is a gradual underprediction of the total-mix which is associated with the reduced width of the left interface (Fig. 12(b)). The insufficient diffuseness of the left interface can be considered as a delayed response to the under-prediction of turbulence kinetic energy production during the past reshocks from $t \approx 2.20 \mathrm{~ms}$ onward. This is particularly evident by the $K$ contour plot at $t=2.70 \mathrm{~ms}$ shown in Fig. 13(b).

An estimate of the time at which the $K$ - $L$ model TKE begins to depart from the iLES data can be obtained by examining Fig. 11. When the self-similar cell Atwood number $\left(A_{S S j}\right)$ becomes much larger than the initial cell Atwood number $\left(A_{0 j}\right)$ in absolute terms, the production term $\left(S_{K}\right)$ becomes excessively large. In terms of 


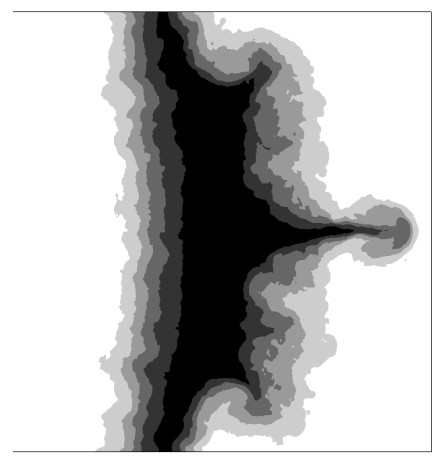

(a) iLES

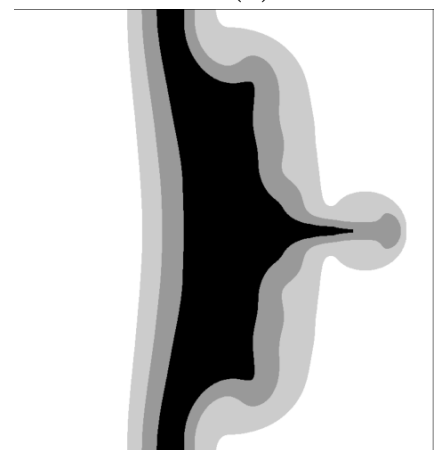

(b) TF

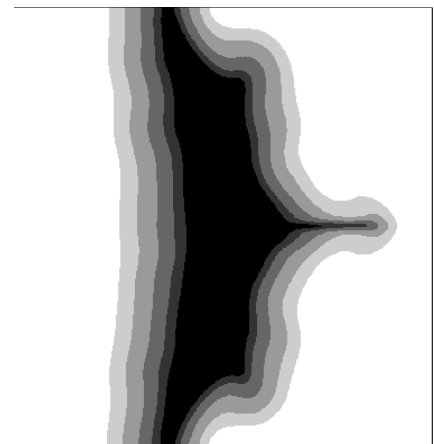

(d) $\mathrm{KL}_{\mathrm{M}}$

(e) $\mathrm{KL}_{\mathrm{M}}$ (Fine)

FIG. 12. Volume fraction, $\tilde{z}$, contour plot at $t=2.70 \mathrm{~ms}$ for the inverse-chevron case, from black $\tilde{z}_{\text {air }}=0.1$ to 0.9 every 0.2 ; (a) iLES, (b) two-fluid model, (c) $K-L$ model - KL variant, (d) $\mathrm{KL}_{\mathrm{M}}$ variant, and (e) $\mathrm{KL}_{\mathrm{M}}$ on fine grid.

magnitude, the initial Atwood number is found to be $\left|A_{0 j}\right| \simeq 0.3$ during shock (for the baseline grid $320 \times 160$ ), gradually decreasing thereafter. The self-similar Atwood number rapidly increases after shock to a larger value $\left|A_{S S j}\right| \simeq 0.5$ (hence the weighting factor $w_{L j}$ introduced in Eq. $(26)^{37}$ ), but in contrast to $A_{0 j}$, it reaches and maintains a peak value of $\left|A_{S S j}\right| \simeq 0.75$. From a physical perspective, the calculation of the Atwood number according to Eq. (26) is strictly valid only while the instability grows in a self-similar fashion. However, this no longer holds true once the interface width collapses due to the reversal of the left interface's travelling direction during the reshock at $t \approx 2.20 \mathrm{~ms}$.

The post-reshock growth rate appears linear in time

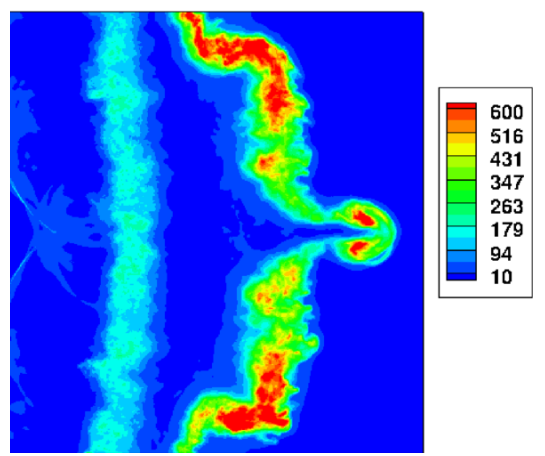

(a) iLES
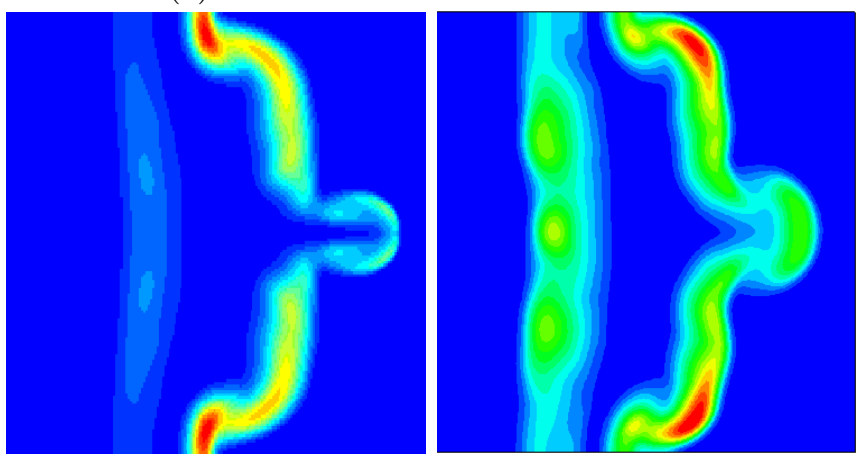

(b) $\mathrm{TF}$

(c) KL
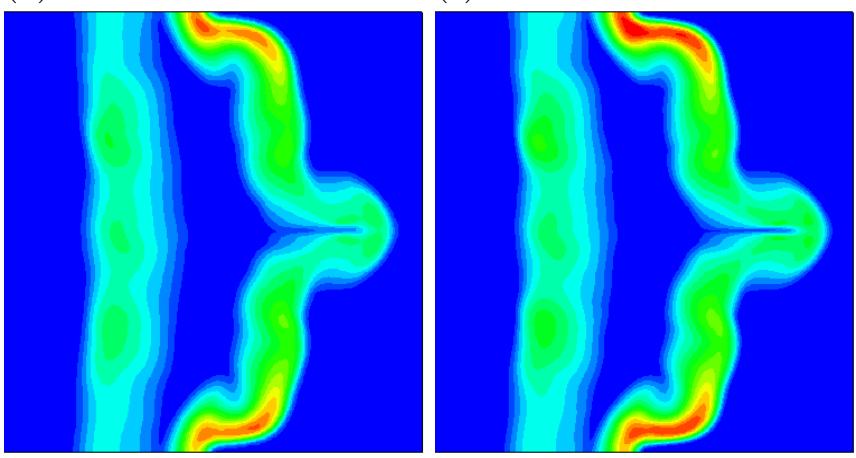

(d) $\mathrm{KL}_{\mathrm{M}}$

(e) $\mathrm{KL}_{\mathrm{M}}$ (Fine)

FIG. 13. Turbulence kinetic energy, $K$, contour plot at $t=$ $2.70 \mathrm{~ms}$ for the inverse-chevron case; (a) iLES, (b) two-fluid model, (c) $K-L$ model - KL variant, (d) $K_{\mathrm{M}}$ variant, and (e) $\mathrm{KL}_{\mathrm{M}}$ on fine grid

only for a short period, leading to the development of a diffuse-interface model ${ }^{47}$. For weak or moderate incident shock strengths, both compressible and vorticityamplification effects are of secondary importance for post-reshock mixing growth. Instead, the dominant term at a diffuse interface is the baroclinic deposition. The same study cautions that numerical confirmation is required when considering different pre-reshock vorticity distributions.

The above prompt to the importance of the self-similar Atwood number term $A_{S S j}$ during reshock in conjunction with the mixing width growth rate. As the initial Atwood number $A_{0 j}$ decreases due to the increasing mix- 


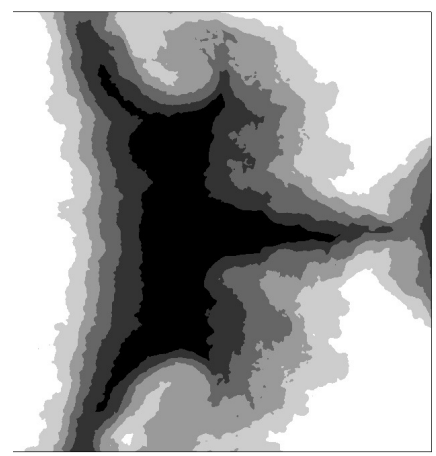

(a) iLES

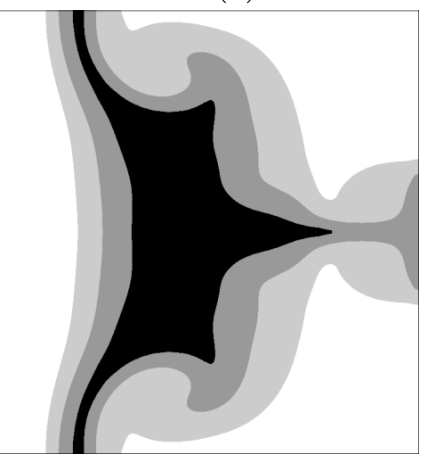

(b) $\mathrm{TF}$

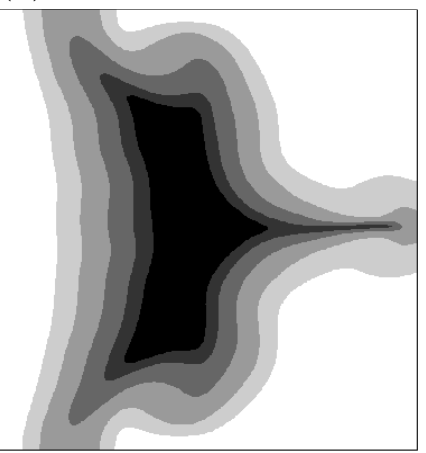

(d) $\mathrm{KL}_{\mathrm{M}}$

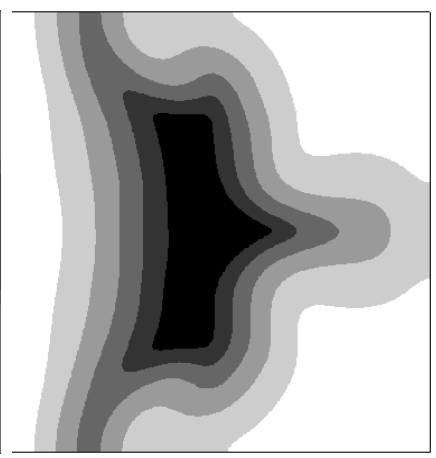

(c) KL

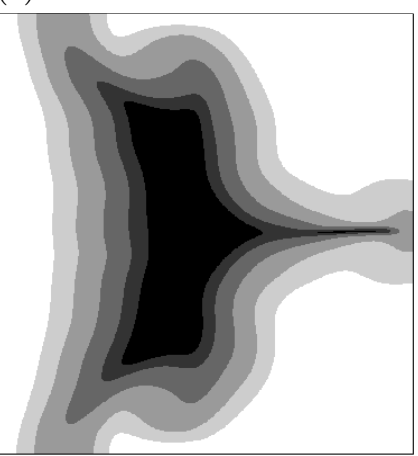

(e) $\mathrm{KL}_{\mathrm{M}}$ (Fine)

FIG. 14. Volume fraction, $\tilde{z}$, contour plot at $t=3.30 \mathrm{~ms}$ for the inverse-chevron case, from black $\tilde{z}_{\text {air }}=0.1$ to 0.9 every 0.2 ; (a) iLES, (b) two-fluid model, (c) $K-L$ model - KL variant, (d) $\mathrm{KL}_{\mathrm{M}}$ variant, and (e) $\mathrm{KL}_{\mathrm{M}}$ on fine grid.

ing width, i.e. the local density difference reduces, $A_{S S j}$ begins to dominate the value of the local Atwood number $\left(A_{L i}\right)$, according to Eq. (26). The density gradient across the interface does not pose an adequate metric for the mixedness of the two fluids. The value of $A_{S S j}$ relates to $A_{0 j}$ as follows: by gradually reducing the production term $S_{K}$ according to Eq. (30) using the ratio of the smallest domain size along a homogeneous direction to the turbulence length scale, $L$, it can help prevent $S_{K}$ from becoming excessively large at late time. Note that except for the compressive term, more advanced turbulence models, such as the second moments BHR model ${ }^{48}$, include further terms in the transport equation of the turbulence length scale that allow for a reduction in $L$.

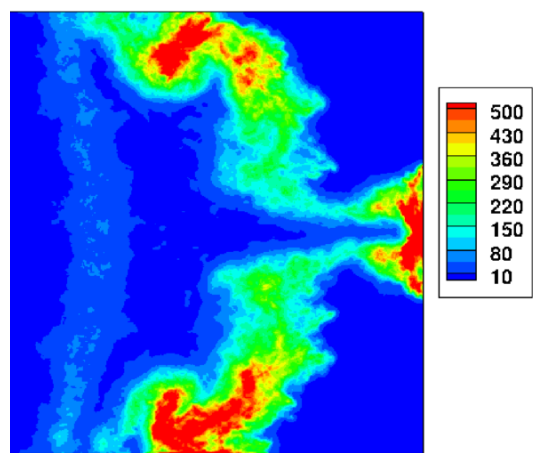

(a) iLES
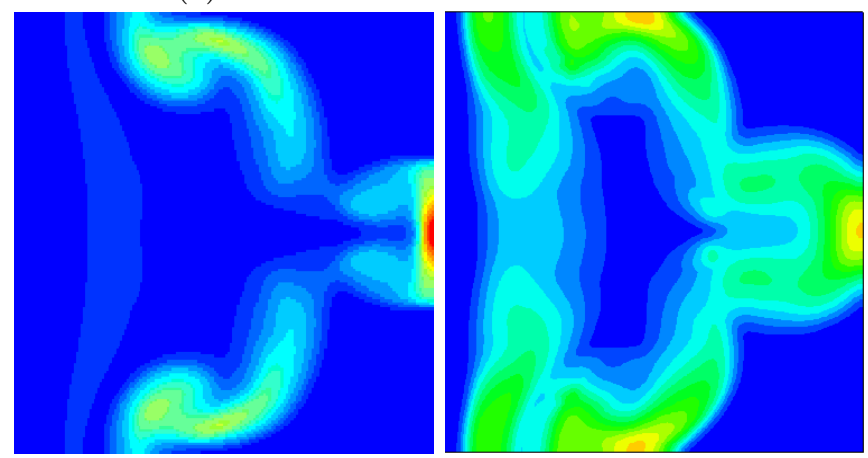

(b) $\mathrm{TF}$

(c) KL
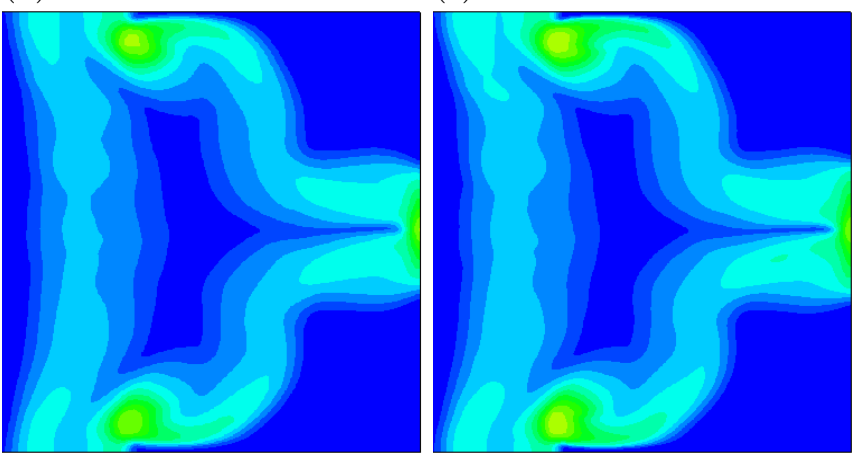

(d) $\mathrm{KL}_{\mathrm{M}}$

(e) $\mathrm{KL}_{\mathrm{M}}$ (Fine)

FIG. 15. Turbulence kinetic energy, $K$, contour plot at $t=$ $3.30 \mathrm{~ms}$ for the inverse-chevron case; (a) iLES, (b) two-fluid model, (c) $K-L$ model - KL variant, (d) $K_{\mathrm{M}}$ variant, and (e) $\mathrm{KL}_{\mathrm{M}}$ on fine grid

Figures 12 and 13 present the volume fraction $(\tilde{z})$ and turbulence kinetic energy $(K \equiv \tilde{k})$ contour plots, respectively, at time $t=2.70 \mathrm{~ms}$. We compare $\mathrm{KL}_{\mathrm{M}}, \mathrm{KL}$ and the TF models against the equivalent 2D Favre-averaged $3 \mathrm{D}$ iLES. The same comparison is also carried out at time $t=3.30 \mathrm{~ms}$ in Figures 14 and 15.

Previous iLES studies ${ }^{25,26}$ have shown that a reshocked layer still exhibits a weakened dependence on the initial conditions. The $K-L$ model $\tilde{z}$ contour plot at time $t=2.70 \mathrm{~ms}$ and particularly the $\mathrm{KL}_{\mathrm{M}}$ variant shown in Fig. 14(d), still exhibits signs of the initial large scale broadband perturbations. This is implied by the waviness of the interfaces at a scale of the order of the domain 
size and is consistent with similar "peaks" and "troughs" noticeable in the spanwise Favre-averaged iLES $\tilde{z}$ contour plot in Fig. 12(a).

The original $K-L$ model (KL variant) behaves very much like the modified variant $\left(\mathrm{KL}_{\mathrm{M}}\right)$ up until the time of the reshocks. The increase of TKE at $t=2.0 \mathrm{~ms}$ is under-predicted, while suddenly at late time $t=3.0 \mathrm{~ms}$ it begins to increase monotonically. As for the doubleplanar case examined previously, this occurs due to the over-estimation of the turbulence kinetic energy production source term $\left(S_{K}\right)$ at the left interface at a late time, further substantiated by Fig. 15(c). The total-mix (MIX) begins to increase monotonically as a result of the overdiffuseness of the left interface in both the double-planar and inverse-chevron test cases as seen in Figs. 4 and 10, respectively. This in turn is caused by the large turbulent viscosity since $\mu_{t} \propto \sqrt{K}$.

Without a mechanism to control the turbulence kinetic energy production at a late time, the KL model grossly overestimates the turbulence kinetic energy at the left interface (Fig. 15(c)). Moreover, the volume fraction $(\tilde{z})$ contours allude to the presence of flow regions afflicted by active, non-physical, isotropic diffusion produced by the KL model variant in sharp contrast to the iLES.

We attribute the diffuseness of the right interface's leading spike to the Boussinesq eddy viscosity assumption, which causes all turbulent diffusion terms (gradient diffusion approximation) to act in an isotropic manner. The above behaviour is particularly obvious while comparing the iLES and $K-L$ model $\tilde{z}$ contour plots in Figs. $12 \mathrm{a}$ and $12 \mathrm{c}$, respectively. The two-dimensional isotropic diffusion limiter $S_{F}$ introduced by Kokkinakis, Drikakis, and Youngs ${ }^{39}$ significantly improves the distribution of the volume fraction, particularly in areas of the flow exhibiting strong two-dimensional motion. Figures 14 and 15 further demonstrate how the turbulent viscosity limiter enables the $K-L$ model to more accurately predict the formation and subsequent impingement of the large scale spike onto the tube's right boundary surface. The TF model shows that, by design, it is not afflicted by the isotropic diffusion problem as the single fluid models are.

Increasing grid resolution has no significant impact on the results obtained. The volume fraction contour plots in Figures 12 and 14 indicate that at the late time the diffusion provided by the turbulence model is much larger than the numerical and largely determines the development of the interface. For the turbulence kinetic energy, the results of Figures 13 and 15 suggest that increasing grid resolution has no significant impact on the magnitude or distribution. The width of the leading spike forming in the middle of the right interface is not affected by the grid resolution either. Any minor differences are attributed to the more substantial numerical diffusion on the coarser grid at the early stage of the development of the interfaces before the turbulence model becomes prevalent.

\section{SUMMARY AND CONCLUSIONS}

We have investigated the $K-L$, and TF turbulence mixing models in two different cases of Richtmyer-Meshkov instability (RMI) induced mixing. We assess the models against equivalent appropriately averaged data obtained using high-resolution iLES.

The first case considered involves the modelling of a double-planar interface, for which we investigate the models' performance in their spatially one-dimensional formulation. Overall the TF model gives better accuracy to the iLES results, particularly at later times where it outperforms the $K-L$ model. The $K-L$ model overpredicts the turbulence kinetic energy at the left interface due to the excessive production of the turbulence kinetic energy source term, $S_{K}$. Using the iLES data for reference, it is elaborated that this discrepancy occurs soon after the initial bubbles and spikes, formed by the first primary shock, collapse during the late time reshocks. The left interface collapse event abruptly alters its selfsimilar growth rate while the resulting enhanced molecular mixing state impedes any subsequent RM instabilities from forming. This behaviour invalidates the $K-L$ model constants, which are determined assuming the instabilities continuously grow in a linear self-similar rate.

We show that an a posteriori threshold mechanism, introduced to limit the production of the turbulence kinetic energy in the $\mathrm{KL}_{\mathrm{M}}$ model variant, mitigates the monotonic increase in the total turbulence kinetic energy (TKE) that occurs during the late time reshocks of the left interface. However, we need to investigate further the modified production term $S_{K}^{L}$ introduced in Eq. (30), because it affects inappropriate regions, e.g. at the right interface. Its shortcomings are due to (i) the lack of information regarding the state of mixedness of the interfaces, and (ii) its heavy reliance on the self-similar Atwood number $\left(A_{S S j}\right)$, which is designed assuming a continuous, uninterrupted self-similar growth. The lack of a term capable of appropriately adjusting the turbulence length scale, $L$, during the collapse of the left interface, unavoidably leads to the over-prediction of the turbulence kinetic energy $(K)$.

Both models manage to perform reasonably well at the right interface, with the more complex TF model maintaining a clear advantage over the $K-L$ model in terms of both volume fraction and turbulence kinetic energy profiles. For the inverse chevron interface, the $K-L$ model reproduces the turbulence kinetic energy contours at $t=2.7 \mathrm{~ms}$ more faithfully than the TF model. However, by $t=3.3 \mathrm{~ms}$, the $K-L$ model shows signs of excessive diffuseness at the left interface as well as the frontal tip of the leading spike formed at the right interface. The $K-L$ model is designed in mind as a simple model able to capture the self-similar growth of RT and RM flows with a planar (extended) interface. It is not intended to capture the highly localized, non-self-similar tip of a chevron for which the comparison is better with the more complex, but also computationally expensive, multi-fluid 
model.

Furthermore, we have investigated an isotropic diffusion control limit mechanism proposed by Kokkinakis, Drikakis, and Youngs ${ }^{39}$ to alleviate the non-physical over-diffuseness caused by linear eddy viscosity models such as the $K-L$ in 2D flow simulations. Originally ${ }^{39}$, only the RTI dominant 2D tilted-rig case was considered. Here, we apply the turbulent viscosity limiter $\left(S_{F}\right)$ to the RMI dominant (some KHI) 2D inverse-chevron test-case. We have found that the limiter improves the volume fraction contours of regions of the flow that exhibit large mean gradients in both spatial directions. For example, at the inclined (2D) interface and at late time $t=3.30 \mathrm{~ms}$, a more natural (iLES-like) shape of the large scale protruding spike as well as lower and upper "rollups" is observed. More crucially, the impingement of the spike onto the right wall boundary surface is now almost as accurately modelled as the TF model.

\section{ACKNOWLEDGMENTS}

Dimitris Drikakis wishes to express his appreciation to the AWE (United Kingdom) for their financial support through the William Penney Fellowship award. The manuscript contains material (c)British Crown Owned Copyright 2020/AWE, reproduced with permission.

Results were obtained using the ARCHIE-WeSt High Performance Computer (www. archie-west.ac.uk) based at the University of Strathclyde. The TF simulations were performed at AWE, Aldermaston, UK.

\section{DATA AVAILABILITY}

The data that support the findings of this study are available on request from the corresponding author. The data are not publicly available due to the funding body restrictions.

\section{REFERENCES}

\footnotetext{
${ }^{1}$ R. Richtmyer, "Taylor instability in shock acceleration of compressible fluids," Communications on Pure and Applied Mathematics 13, 297-319 (1960).

${ }^{2}$ E. E. Meshkov, "Instability of the interface of two gases accelerated by a shock wave," Fluid Dynamics 4, 101-104 (1969).

${ }^{3}$ R. Holmes, G. Dimonte, B. Fryzell, M. Gittings, J. Grove, M. Schneider, D. Sharp, A. Velikovich, R. Weaver, and Q. Zhang, "Richtmyer-Meshkov instability growth: experiment, simulation and theory," Journal of Fluid Mechanics 389, 55-79 (1999).

${ }^{4}$ P. Amendt, J. D. Colvin, R. E. Tipton, D. E. Hinkel, M. J. Edwards, O. L. Landen, J. D. Ramshaw, L. J. Suter, W. S. Varnum, and R. G. Watt, "Indirect-drive noncryogenic double-shell ignition targets for the National Ignition Facility: Design and analysis," Physics of Plasmas 9, 2221-2233 (2002).

${ }^{5}$ M. Brouillette, "The Richtmyer-Meshkov Instability," Annual Review of Fluid Mechanics 34, 445-468 (2002).
}

${ }^{6}$ J. Yang, T. Kubota, and E. E. Zukoski, "Applications of shockinduced mixing to supersonic combustion," AIAA Journal 31, 854-862 (1993).

${ }^{7}$ W. D. Arnett, J. N. Bahcall, R. P. Kirshner, and S. E. Woosley, "Supernova 1987a," Annual Review of Astronomy and Astrophysics 27, 629-700 (1989).

${ }^{8}$ A. S. Almgren, J. B. Bell, C. A. Rendleman, and M. Zingale, "Low mach number modeling of Type Ia supernovae. I. Hydrodynamics," Astrophysical Journal Letters 637, 922-936 (2006).

${ }^{9}$ U. Alon, J. Hecht, D. Ofer, and D. Shvarts, "Power laws and similarity of Rayleigh-Taylor and Richtmyer-Meshkov mixing fronts at all density ratios," Physical Review Letters 74, 534537 (1995).

${ }^{10}$ J. D. Ramshaw, "Simple model for linear and nonlinear mixing at unstable fluid interfaces with variable acceleration," Physical Review E 58, 5834-5840 (1998).

${ }^{11}$ B. Cheng, J. Glimm, and D. H. Sharp, "A three-dimensional renormalization group bubble merger model for Rayleigh-Taylor mixing," Chaos: An Interdisciplinary Journal of Nonlinear Science 12, 267-274 (2002).

${ }^{12}$ G. Dimonte and M. Schneider, "Density ratio dependence of Rayleigh-Taylor mixing for sustained and impulsive acceleration histories," Physics of Fluids 12, 304-321 (2000).

${ }^{13}$ D. L. Youngs, "Modelling turbulent mixing by Rayleigh-Taylor instability," Physica D: Nonlinear Phenomena 37, 270-287 (1989).

${ }^{14}$ D. L. Youngs, "Numerical simulation of mixing by RayleighTaylor and Richtmyer-Meshkov instabilities," Laser and Particle Beams 12, 725-750 (1994).

${ }^{15}$ Y. Chen, J. Glimm, D. H. Sharp, and Q. Zhang, "A two-phase flow model of the Rayleigh-Taylor mixing zone," Physics of Fluids 8, 816-825 (1996).

${ }^{16}$ A. Llor, Statistical Hydrodynamic Models for Developing Mixing Instability Flows (Springer, 2005).

${ }^{17}$ S. Gauthier and M. Bonnet, "A k- $\epsilon$ model for turbulent mixing in shock-tube flows induced by Rayleigh-Taylor instability," Physics of Fluids A: Fluid Dynamics 2, 1685-1694 (1990).

${ }^{18}$ J. T. Morán-López and O. Schilling, "Multicomponent Reynoldsaveraged Navier-Stokes simulations of reshocked RichtmyerMeshkov instability-induced mixing," High Energy Density Physics 9, 112 - 121 (2013).

${ }^{19} \mathrm{G}$. Dimonte and R. Tipton, "K-L turbulence model for the selfsimilar growth of the Rayleigh-Taylor and Richtmyer-Meshkov instabilities," Physics of Fluids 18, 085101 (2006).

${ }^{20}$ V. P. Chiravalle, "The K-L turbulence model for describing buoyancy-driven fluid instabilities," Laser and Particle Beams 24, 381-394 (2006).

${ }^{21} \mathrm{D}$. L. Youngs, "Representation of the molecular mixing process in a two-phase flow turbulent mixing model," in Proceedings of the 5th International Workshop on the Physics of Compressible Turbulent Mixing (World Scientific, 1995) pp. 83-88.

${ }^{22}$ D. A. Holder and C. J. Barton, "Shock tube Richtmyer-Meshkov experiments: Inverse chevron and half height," in Proceedings of the Ninth International Workshop on the Physics of Compressible Turbulent Mixing, edited by S. B. Dalziel (University of Cambridge, 2004).

${ }^{23}$ M. Hahn, D. Drikakis, D. L. Youngs, and R. J. R. Williams, "Richtmyer-Meshkov turbulent mixing arising from an inclined material interface with realistic surface perturbations and reshocked flow," Physics of Fluids 23, 046101 (2011).

${ }^{24}$ I. W. Kokkinakis, D. Drikakis, and D. L. Youngs, "Vortex morphology in richtmyer-meshkov-induced turbulent mixing," Physica D: Nonlinear Phenomena 407, 132459 (2020).

${ }^{25}$ B. Thornber, D. Drikakis, D. L. Youngs, and R. J. R. Williams, "Growth of a Richtmyer-Meshkov turbulent layer after reshock," Physics of Fluids 23, 095107 (2011).

${ }^{26}$ B. Thornber, D. Drikakis, D. L. Youngs, and R. J. R. Williams, "The influence of initial conditions on turbulent mixing due to Richtmyer-Meshkov instability," Journal of Fluid Mechanics 654, 99-139 (2010). 
${ }^{27}$ P. E. Dimotakis, "The mixing transition in turbulent flows," Journal of Fluid Mechanics 409, 69-98 (2000).

${ }^{28}$ A. W. Cook and P. E. Dimotakis, "Transition stages of RayleighTaylor instability between miscible fluids," Journal of Fluid Mechanics 457, 410-411 (2002).

${ }^{29}$ G. Allaire, S. Clerc, and S. Kokh, "A five-equation model for the simulation of interfaces between compressible fluids," Journal of Computational Physics 181, 577-616 (2002).

${ }^{30}$ A. W. Cook, "Enthalpy diffusion in multicomponent flows," Physics of Fluids 21, 055109 (2009).

${ }^{31}$ S. K. Godunov, "A difference method for numerical calculation of discontinuous solutions of the equations of hydrodynamics," Matematičeskij sbornik 47(89), 271-306 (1959).

${ }^{32}$ E. F. Toro, M. Spruce, and W. Speares, "Restoration of the contact surface in the HLL-Riemann solver," Shock Waves 4, 25-34 (1994).

${ }^{33}$ B. V. Leer, "Towards the ultimate conservative difference scheme. IV. A new approach to numerical convection," Journal of Computational Physics 23, 276-299 (1977).

${ }^{34}$ K. H. Kim and C. Kim, "Accurate, efficient and monotonic numerical methods for multi-dimensional compressible flows: Part II: Multi-dimensional limiting process," Journal of Computational Physics 208, $570-615$ (2005).

${ }^{35}$ B. Thornber, A. Mosedale, D. Drikakis, D. Youngs, and R. J. R. Williams, "An improved reconstruction method for compressible flows with low Mach number features," Journal of Computational Physics 227, 4873-4894 (2008).

${ }^{36}$ S. Gottlieb and C.-W. Shu, "Total variation diminishing RungeKutta schemes," Mathematics of Computation 67, 73-85 (1998).

${ }^{37}$ I. W. Kokkinakis, D. Drikakis, D. L. Youngs, and R. J. R. Williams, "Two-equation and multi-fluid turbulence models for Rayleigh-Taylor mixing," International Journal of Heat and Fluid Flow 56, 233 - 250 (2015).

${ }^{38}$ B. Vreman, B. Geurts, and H. Kuerten, "Realizability conditions for the turbulent stress tensor in large-eddy simulation," Journal of Fluid Mechanics 278, 351-362 (1994).

${ }^{39}$ I. W. Kokkinakis, D. Drikakis, and D. L. Youngs, "Modeling of Rayleigh-Taylor mixing using single-fluid models," Phys. Rev. E
99, 013104 (2019).

${ }^{40}$ D. L. Youngs, "Three-dimensional numerical simulation of turbulent mixing by Rayleigh-Taylor instability," Physics of Fluids A: Fluid Dynamics 3, 1312-1320 (1991).

${ }^{41}$ J. C. V. Hansom, P. A. Rosen, T. J. Goldack, K. Oades, P. Fieldhouse, N. Cowperthwaite, D. L. Youngs, N. Mawhinney, and A. J. Baxter, "Radiation driven planar foil instability and mix experiments at the AWE HELEN laser," Laser and Particle Beams 8, 51-71 (1990).

${ }^{42}$ B. M. Haines, F. F. Grinstein, and J. D. Schwarzkopf, "Reynolds-averaged Navier-Stokes initialization and benchmarking in shock-driven turbulent mixing," Journal of Turbulence $\mathbf{1 4}$ 46-70 (2013).

${ }^{43}$ F. F. Grinstein, "Initial conditions and modeling for simulations of shock driven turbulent material mixing," Computers \& Fluids 151, 58 - 72 (2017), a special issue in honor of Cecil "Chuck" E. Leith.

${ }^{44}$ B. Thornber, "Impact of domain size and statistical errors in simulations of homogeneous decaying turbulence and the RichtmyerMeshkov instability," Physics of Fluids 28, 045106 (2016).

${ }^{45}$ D. Besnard, F. H. Harlow, R. M. Rauenzahn, and C. Zemach, "Turbulence transport equations for variable-density turbulence and their relationship to two-field models," Tech. Rep. LA-UR12303 (Los Alamos National Lab., NM (United States), 1992).

${ }^{46}$ A. Banerjee, R. A. Gore, and M. J. Andrews, "Development and validation of a turbulent-mix model for variable-density and compressible flows," Physical Review E 82, 046309 (2010).

${ }^{47}$ M. Lombardini, D. J. Hill, D. I. Pullin, and D. I. Meiron, "Atwood ratio dependence of Richtmyer-Meshkov flows under reshock conditions using large-eddy simulations," Journal of Fluid Mechanics 670, 439-480 (2011).

${ }^{48}$ J. D. Schwarzkopf, D. Livescu, R. A. Gore, R. M. Rauenzahn, and J. R. Ristorcelli, "Application of a second-moment closure model to mixing processes involving multicomponent miscible fluids," Journal of Turbulence 12, N49 (2011). 